\title{
An analytical study of Rossby waves in a zonally-varying basic flow
}

\section{Mariam Tariri}

\author{
A thesis submitted to \\ the Faculty of Graduate Studies and Research \\ in partial fulfillment of \\ the requirements for the degree of
}

\author{
Master of Science \\ School of Mathematics and Statistics \\ Ottawa-Carleton Institute of Mathematics and Statistics \\ Carleton University \\ Ottawa, Ontario, Canada \\ May, 2007 \\ (C) Copyright 2007 \\ Mariam Tariri
}




$\begin{array}{ll}\begin{array}{l}\text { Library and } \\ \text { Archives Canada }\end{array} & \begin{array}{l}\text { Bibliothèque et } \\ \text { Archives Canada }\end{array} \\ \begin{array}{l}\text { Published Heritage } \\ \text { Branch }\end{array} & \begin{array}{l}\text { Direction du } \\ \text { Patrimoine de l'édition }\end{array} \\ \begin{array}{l}\text { 395 Wellington Street } \\ \text { Ottawa ON K1A ON4 }\end{array} & \begin{array}{l}\text { 395, rue Wellington } \\ \text { Ottawa ON K1A ON4 } \\ \text { Canada }\end{array}\end{array}$

Your file Votre référence ISBN: 978-0-494-27025-7 Our file Notre référence ISBN: 978-0-494-27025-7

NOTICE:

The author has granted a nonexclusive license allowing Library and Archives Canada to reproduce, publish, archive, preserve, conserve, communicate to the public by telecommunication or on the Internet, loan, distribute and sell theses worldwide, for commercial or noncommercial purposes, in microform, paper, electronic and/or any other formats.

The author retains copyright ownership and moral rights in this thesis. Neither the thesis nor substantial extracts from it may be printed or otherwise reproduced without the author's permission.
AVIS:

L'auteur a accordé une licence non exclusive permettant à la Bibliothèque et Archives Canada de reproduire, publier, archiver, sauvegarder, conserver, transmettre au public par télécommunication ou par l'Internet, prêter, distribuer et vendre des thèses partout dans le monde, à des fins commerciales ou autres, sur support microforme, papier, électronique et/ou autres formats.

L'auteur conserve la propriété du droit d'auteur et des droits moraux qui protège cette thèse. $\mathrm{Ni}$ la thèse ni des extraits substantiels de celle-ci ne doivent être imprimés ou autrement reproduits sans son autorisation.
In compliance with the Canadian

Privacy Act some supporting forms may have been removed from this thesis.

While these forms may be included in the document page count, their removal does not represent any loss of content from the thesis.
Conformément à la loi canadienne sur la protection de la vie privée, quelques formulaires secondaires ont été enlevés de cette thèse.

Bien que ces formulaires aient inclus dans la pagination, il n'y aura aucun contenu manquant.

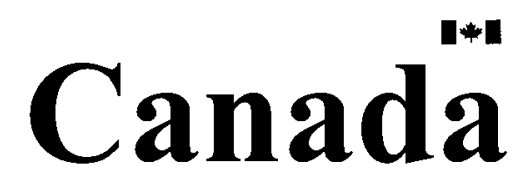




\section{Abstract}

This thesis describes a mathematical study of the evolution of Rossby waves in the atmosphere. These are large scale waves that result from the Earth's rotation. Analytical methods including Laplace transforms, multiple scaling and asymptotic expansions are used to obtain approximate solutions that describe the propagation of the waves. Each fluid variable in the governing equation is written as the sum of a basic steady term and a time dependent perturbation. In previous related studies the basic flow was considered to be a function of the latitude only, but in this thesis we study a configuration where the basic flow varies slowly with respect to the zonal variable. This is a more realistic representation of the general circulation of the real atmosphere. The governing equation includes these small parameters and depending on the relative magnitude of these parameters the problem can be linear or nonlinear. We consider the scalings that lead to linear problems and derive an approximate solution in each case. These approximate solutions describe interactions between the waves and the basic flow. 


\section{To my mom's memory, Fakhrieh Sabeh my dear dad, Mohammadali Tariri and my sisters Nawal, Ghazal, Amal}




\section{Acknowledgements}

I would like to express my sincere gratitude to my supervisor, Dr. Lucy Campbell for her time and patience, her kindness and support and also her encouragement during the preparation of my thesis.

I would like to thank my family, especially my dear parents for their support and encouragement which guided me through this project as well as many other important achievements that I have experienced in my life.

Finally I want to thank my fiancé Momen Mokhtari for his moral support throughout this project. He was always there for me through difficult times throughout the course of my project. 


\section{Contents}

Abstract

$\begin{array}{ll}\text { Dedications } & \text { ii }\end{array}$

$\begin{array}{ll}\text { Acknowledgements } & \text { iii }\end{array}$

Contents iv

List of Figures $\quad$ viii

1 Introduction 1

2 The governing equations $\quad 8$

2.1 Derivation of the Governing Equations $\ldots \ldots \ldots \ldots$

2.1 .1 Continuity Equation $\ldots \ldots \ldots \ldots$

2.1 .2 Momentum Equation . . . . . . . . . . . . . . . . . 11

2.1 .3 Energy Equation $\ldots \ldots \ldots \ldots \ldots$

2.2 Streamfunction and vorticity $\ldots \ldots \ldots \ldots \ldots \ldots$

2.3 The Coriolis force and the $\beta$-plane approximation $\ldots \ldots \ldots 17$

iv 
3 Geophysical flows $\quad 19$

3.1 Nondimensionalization . . . . . . . . . . . . . . 19

3.2 Critical layer . . . . . . . . . . . . . . . 21

4 Formulation of the problem for a zonally-varying basic flow $\quad 27$

4.1 Derivation of the equation ................ 27

4.2 Linear scalings . . . . . . . . . . . . . . . . . 32

4.3 Solution of the $\mathrm{O}(1)$ equation $\ldots \ldots \ldots . \ldots . \ldots 34$

5 Derivation of the linear solutions $\quad \mathbf{4 0}$

5.1 The zonally-varying flow . . . . . . . . . . . . . . . 40

5.2 The $O(\mu)$ term . . . . . . . . . . . . . . . . . . 41

5.3 Case $1 \ldots \ldots \ldots \ldots \ldots$. . . . . . . . . . . . . . 42

5.4 Case $2 \ldots \ldots \ldots \ldots \ldots \ldots \ldots$

5.5 Late time solution . . . . . . . . . . . . . . . . 50

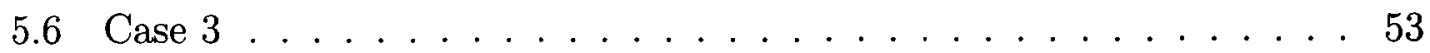

5.7 The critical layer thickness . . . . . . . . . . . . . . 56

5.8 Inner solution . . . . . . . . . . . . . . 58

6 Conclusions $\quad 61$

A Evaluation of the contributions from the branch points in case 164

B Solution of the non-homogeneous equation in case $1 \quad 70$

B.1 The case $B^{\prime}(y)=$ a constant . . . . . . . . . . . . . 73

C Evaluation of the solution for $|y| t \sim O(1) \quad 77$ 
Bibliography

81

vi

Reproduced with permission of the copyright owner. Further reproduction prohibited without permission. 


\section{List of Figures}

5.1 Time regime comparison $\ldots \ldots \ldots \ldots \ldots$

A.1 Contour of integration in the complex $s$-plane for equation (4.17). The branch points $s=-i k y_{1}$ and $s=-i k y$ are indicated by the black dots. . . . . . . . . . . . . . . . . . . . . 69 


\section{Chapter 1}

\section{Introduction}

The study of the stability and propagation of waves in hydrodynamic flows has received a lot attention in the past century. Research on hydrodynamic stability has been motivated by the goal of understanding the transition from laminar (smooth and ordered) flow to turbulent (disordered) flow. Hydrodynamic stability theory is used to predict the behavior of the fluid flow before the onset of turbulence. In studying wave propagation, waves are generally considered to be perturbations on some basic flow. In linear stability theory, the wave amplitudes are assumed to be small enough so that the governing equations can be linearized with respect to perturbations. With larger wave amplitudes the problems are nonlinear, and interactions between waves and the basic flow can be studied. These nonlinear interactions sometimes lead to wave breaking and eventually to turbulence. Once the fluid flow becomes turbulent, special "turbulence models" are required (see any book on fluid dynamics such as Kundu and Cohen, 2002).

In studying waves in laminar flows it is often assumed that the basic flow is a 
parallel shear flow. In rectangular coordinates $x$ and $y$, a parallel flow is one that has a velocity component in only one coordinate direction e.g. a flow with velocity $(\bar{U}, 0)$. The parallel flow is a shear flow if the velocity varies in the direction of the other coordinate, e.g., $(\bar{u}(y), 0)$. To study wave propagation in a parallel shear flow the fluid velocity is written as the sum of the basic velocity $(\bar{u}(y), 0)$ and the perturbation $\left(u^{\prime}, v^{\prime}\right)$. The velocity is usually taken to be the initial mean in the $x$-direction, with the mean being calculated over a wavelength in the $x$-direction. Equations for the perturbations are obtained, and since $\bar{u}(y)$ is specified one can solve for $u^{\prime}$ and $v^{\prime}$.

One situation that can lead to wave-mean flow interactions occurs if the mean velocity $\bar{u}(y)$ is equal to the phase speed of the waves at some value of $y$. This value of $y$ is called a critical line and it corresponds to a singularity in the linear equation for the perturbations. The singularity arises due to the assumptions that are made in simplifying the equations: that the equations are linear and there is no viscosity (friction). In order to obtain solutions near the singularity, a "critical layer" is defined around the critical line and either the viscous terms (Lin, 1966) or the nonlinear terms (Benney and Bergeron, 1969, Davis, 1969) are re-introduced to the equations within the critical layer, in order to remove the singularity. The procedure is analogous to that used to study boundary layers in fluid flows near solid boundaries. Once a solution is obtained in the critical layer it can be matched to the outer solution, i.e., the linear inviscid solution away from the critical line.

An alternative procedure for obtaining a solution in a shear flow with a critical layer is to allow the amplitude of the perturbations to be time dependent and thus remove the singularity from the linear inviscid equations. This method was used by 
Dickinson (1970) and Warn and Warn (1976) to study the time evolution of Rossby waves propagating in the north-south direction in a horizontal plane. Rossby waves are planetary-scale waves that occur in the atmosphere and result from the effects of the rotation of the Earth, i.e., the Coriolis force.

In the time dependent problem studied by Dickinson (1970) and Warn and Warn (1976), the waves are forced by specifying a boundary condition periodic in the zonal or longitudinal $(x)$ direction at some fixed latitude $(y)$ corresponding to the northern boundary at some rectangular region on a horizontal plane. As a result of the boundary condition a Rossby wave periodic in $x$ direction and in time propagates southwards towards the critical line or the critical latitude. The solution can be obtained by taking a Laplace transform in the time variable. The solution shows that the wave amplitude is reduced to zero at the critical layer. The momentum flux of the waves is also reduced to zero, i.e., there is a jump in the wave momentum flux from one side of critical layer to the other. The physical interpretation of this discontinuity is that the waves are absorbed by the mean flow in the critical layer. However since the equations are linear the waves can not actually affect the mean flow.

In the nonlinear version of this problem (Stewartson 1978, Warn and Warn 1978) an approximate analytic solution in the form of asymptotic series can be found. Since the equations are nonlinear, the interaction between the waves and mean flow can be studied. The solution shows that the wave momentum flux goes to zero at the critical layer and the mean flow $\bar{u}(y)$ is modified accordingly in the critical layer. The physical interpretation of these changes is that the waves are absorbed by the mean flow, and as a result the mean flow is accelerated. In the nonlinear 
problem this absorption of the waves occurs at early time. However, the nonlinear terms in the equation grow with time, and eventually they become the same order of magnitude as the linear terms. At late time the solution shows that the jump in the momentum flux at the critical layer becomes zero and eventually changes sign. This means that the waves are being reflected by the mean flow. Numerical solutions show that at late time the flow alternates between a state in which the waves are absorbed by the mean flow and one in which they are reflected (Béland, 1978, Campbell, 2004). Eventually, if the wave amplitudes are large enough, they overturn and break.

Critical layer phenomena are of great importance in the study of waves in geophysical flows, i.e., in the atmosphere and ocean, because the wave absorption and reflection that occur in critical layers lead to changes in the general circulation. Critical layers occur not only for Rossby waves but also for internal gravity waves, the small scale waves that result from the density stratification and buoyancy of the atmosphere and ocean. Booker and Bretheton (1967) derived analytic solutions for linear gravity waves in the prescence of a critical layer and showed that gravity waves are also absorbed at their critical layers.

Brown and Stewartson's (1982) analytic studies of the corresponding nonlinear problem show that gravity wave reflections can occur at late time. More recently nonlinear numerical simulations of Campbell and Maslowe (2003) show that at late time the flow alternates between absorption and reflection in a similar manner to that of the Rossby wave critical layer.

Critical layer theories may help in our understanding of certain phenomena observed in the atmosphere and ocean, for example, the quasi-biennial oscillation in the 
equatorial stratosphere (Lindzen and Holton, 1968), stratospheric sudden warming in the northern hemisphere (Matsuno, 1971) and the dynamics of the southern ocean (Hughes, 1996). Observations of wave motion in the atmosphere illustrate critical layer absorption, for example (Holton, 1975, Fig 1.9, Hare, 1968), and critical layer reflection (Molteni et al, Brunet and Haynes, 1996).

The configuration studied by Stewartson (1978) and Warn and Warn (1978) in which the basic flow velocity is $(\bar{u}(y), 0)$ is a reasonable approximation for the flow in the stratosphere, i.e., the region in the atmosphere above $50 \mathrm{~km}$. In the stratosphere the basic flow is more or less zonally uniform, i.e., it varies with latitude $y$ but is independent of $x$, the zonal coordinate. However, Enomoto and Matsuda (1999) point out that in the troposphere, the lower region of the atmosphere, the zonal variations of the basic flow are generally not negligible and thus a more realistic basic flow in that region would be one that varies in the $x$ direction as well as $y$. Based on this observation, Enomoto and Matsuda carried out a numerical investigation of Rossby wave propagation in the presence of a critical layer in a zonally-varying basic flow. The basic flow was assumed to consist of a zonally-uniform and a zonallyvarying component. A different reflection mechanism was found in their results. Because of the zonal variation in the flow, reflection could take place even in the linear problem where there was no wave induced mean flow.

Enomoto and Matsuda's numerical study is the motivation for the project described in this thesis. The goal of the thesis is to understand and more completely describe the critical layer behavior in the configuration where the basic flow varies in the zonal direction. In particular, we are interested in finding out whether there can be critical layer reflection of the waves in a linearized problem. In our inves- 
tigation we shall use analytic techniques, Laplace transform, perturbation theory, asymptotic expansions and multiple scaling, to derive approximate solutions for this configuration. In our formulation there are three small parameters, which describe the amplitude of the waves relative to the basic flow, the magnitude of the zonallyvarying component of the basic flow relative to the zonally-uniform part, and the zonal extent of the zonally-varying component. Each of these parameters could be chosen based on typical values in the real atmosphere. Depending on the relative magnitude of the three parameters, the problem can be either linear or nonlinear at leading order. We shall derive solutions for three of the linear configurations.

Our configuration differs from that of Enomoto and Matsuda in several respects, the most important being that we solve two-dimensional equations in a rectangular region in a horizontal plane while their model is based on the spherical coordinates. In spite of the differences in the model formulation we shall see that our conclusions are in qualitative agreement with theirs. In particular, we shall see that with a zonally-varying basic flow it is possible to have wave reflections at the critical layer even when the equations are linear, which is consistent with their conclusions.

An overview of the thesis is as follows: in chapter 2 we give a brief introduction to the equations of fluid dynamics and then describe the assumptions that lead to a governing equation that describes the propagation of forced Rossby waves in a two-dimensional horizontal plane. We also introduce and define some basic concepts that will be needed to clearly define our problem. In chapter 3 we describe the formulation that we shall use to study the zonally-varying basic flow. The equations are written in non-dimensional form and the three relevant parameters are defined. Chapter 4 describes the analytic procedure used to obtain approximate linear solu- 
tions. Finally, in chapter 5 we discuss these solutions and give some suggestions for further related projects, including a discussion of the nonlinear configurations. 


\section{Chapter 2}

\section{The governing equations}

\subsection{Derivation of the Governing Equations}

In this chapter we shall give a brief overview of the derivation of the governing equations for fluid flow and the assumptions that are made to obtain simplified equations describing the propagation of Rossby waves on a horizontal plane. To derive these equations we consider fluid motion in some region in space and time defined by a spatial variable $\mathbf{x}$ and time $t$. The vector $\mathbf{x}$ could be defined in any coordinate system. Throughout this thesis we shall use rectangular Cartesian coordinates so $\mathbf{x}=(x, y, z)$ where $x, y$ and $z$ are the independent variables defined in the direction of longitude, latitude and altitude respectively. The dependent variables in the equations are the fluid properties, e.g. pressure $p$, density $\rho$, temperature $T$ and velocity $\mathbf{u}=(u, v, w)$.

First we need to introduce some basic concepts. An important concept is that of frame of reference. There are two types of reference frames for fluid motion, 
the Eulerian and the Lagrangian. In the Eulerian frame the fluid properties are measured relative to the fixed coordinate axes. In the Lagrangian frame the fluid properties are measured following the fluid motion which means that if we study fluid within a control volume it moves with the fluid and thus always contains the same fluid particles. In general, the Lagrangian frame is preferred for deriving conservation laws. However, the Eulerian frame is more convenient for the present context and we shall use it here. In the Eulerian frame we introduce a special notation to define the rate of change of a property following the fluid motion. This rate of change is called the total derivative and is represented by the notation $\frac{D}{D t}$. The total derivative is given by $\frac{D}{D t}=\frac{\partial}{\partial t}+\mathbf{u} \cdot \nabla$, where $\frac{\partial}{\partial t}$ is the ratio of change of the property in the fixed Eulerian frame with respect to time.

The governing equations for fluid motion are based on conservation laws for mass, momentum and energy which we shall describe in the following sections. A detailed derivation is given in fluid dynamics texts such as Kundu and Cohen (2002). In addition the state of a fluid can be specified by a relationship between pressure, temperature and density which is called the equation of state. For example, for a so-called perfect or ideal fluid the equation of state is $p=\rho R T$ where $R$ is the ideal gas constant, $p$ is the pressure and $T$ is the temperature. Dry air in the atmosphere can be considered as an ideal gas so this equation is valid for atmospheric flows.

\subsubsection{Continuity Equation}

According to the basic principles of physics, matter can neither be created nor destroyed so the mass of a closed system does not change with time. In the context 
of fluid motion, the rate of mass flow into any volume $V$ must be equal to the rate of change of mass in $V$. To put this concept in mathematical terms, let us consider a fixed volume $V$, bounded by a closed surface defined in terms of rectangular coordinates $x, y, z$ in three-dimensions. Then the rate of change of mass in $V$ is given by

$$
\frac{d}{d t} \int_{V} \rho \mathrm{d} V=\int_{V} \frac{\partial \rho}{\partial t} \mathrm{~d} V
$$

where $\rho(x, y, z, t)$ is the density. Also the rate of mass flow across the volume is

$$
\int_{A} \rho \mathbf{u} \cdot \mathrm{d} \mathbf{A}
$$

where $d \mathbf{A}=\hat{\mathbf{n}} d A$ with $\hat{\mathbf{n}}$ being the unit outward normal vector to the surface and the scalar $d A$ being an area element on the surface $A$ of the volume $V$. So conservation of mass requires that:

$$
\int_{V} \frac{\partial \rho}{\partial t} \mathrm{~d} V=-\int_{A} \rho \mathbf{u} \cdot \mathrm{d} \mathbf{A}
$$

By the divergence theorem,

$$
\int_{A} \rho \mathbf{u} \cdot \mathrm{d} \mathbf{A}=\int_{\mathbf{V}} \nabla \cdot(\rho \mathbf{u}) \mathrm{d} \mathbf{V}
$$

Putting (2.1) and (2.2) together gives

$$
\int_{V}\left[\frac{\partial \rho}{\partial t}+\nabla \cdot(\rho \mathbf{u})\right] \mathrm{d} V=0
$$

This relation is valid for an arbitrary volume, therefore the integrand should be zero at every point since $\rho$ and $\mathbf{u}$ are assumed to be continuous functions. The differential 
equation for conservation of mass is

$$
\frac{\partial \rho}{\partial t}+\nabla \cdot(\rho \mathbf{u})=\mathbf{0}
$$

This equation is referred to as the continuity equation. It should be stated that for an incompressible fluid for which $\rho$ does not change with pressure, the continuity equation simply becomes $\nabla \cdot \mathbf{u}=\mathbf{0}$.

\subsubsection{Momentum Equation}

Newton's second law of motion states that the rate of change of momentum of an object is equal to the sum of forces acting on it. This can be written as

$$
\frac{D}{D t}(M \mathbf{u})=\sum \mathbf{F}
$$

where $M$ is the mass and $\mathbf{F}$ represents forces acting on the system. For a fluid these forces could included the forces resulting from viscosity, gravity, compression or expansion, i.e., pressure, and in the case of geophysical flows, the Coriolis force which results from the rotation of the Earth. For an incompressible rotating fluid the equations for conservation of momentum in three-dimensional rectangular coor- 
dinates are:

$$
\begin{aligned}
\rho\left(\frac{D u}{D t}+f_{*} w-f v\right) & =-\frac{\partial p}{\partial x}+\frac{\partial \tau^{x x}}{\partial x}+\frac{\partial \tau^{x y}}{\partial y}+\frac{\partial \tau^{x z}}{\partial z} \\
\rho\left(\frac{D v}{D t}+f u\right) & =-\frac{\partial p}{\partial y}+\frac{\partial \tau^{x y}}{\partial x}+\frac{\partial \tau^{y y}}{\partial y}+\frac{\partial \tau^{y z}}{\partial z} \\
\rho\left(\frac{D w}{D t}-f_{*} u\right) & =-\frac{\partial p}{\partial z}-\rho g+\frac{\partial \tau^{x z}}{\partial x}+\frac{\partial \tau^{y z}}{\partial y}+\frac{\partial \tau^{z z}}{\partial z}
\end{aligned}
$$

where $f=2 \Omega \sin \phi$ is the Coriolis parameter and $f_{*}=2 \Omega \cos \phi$ is the reciprocal Coriolis parameter, $\Omega$ is the earth's angular velocity and $\phi$ is the latitude, $\rho$ is the density, $p$ is the pressure, $g$ is the acceleration due to gravity, and the $\tau$ terms are normal and shear stresses due to viscosity. Processes of internal friction occur when different fluid particles move with different velocities so that there is relative motion between various parts of the fluid. Thus, the stress components can be expressed in terms of velocity gradients, and if the velocity gradients are assumed to be small the stress can be considered to be a linear function of the first derivatives only. These assumptions define a Newtonian fluid. The additional assumption that the fluid is isotropic, i.e., homogeneous, leads to the following expressions for the components of the stress.

$$
\begin{aligned}
& \tau^{x x}=\mu\left(\frac{\partial u}{\partial x}+\frac{\partial u}{\partial x}\right), \quad \tau^{y y}=\mu\left(\frac{\partial v}{\partial y}+\frac{\partial v}{\partial y}\right) \\
& \tau^{z z}=\mu\left(\frac{\partial w}{\partial z}+\frac{\partial w}{\partial z}\right), \quad \tau^{x y}=\mu\left(\frac{\partial u}{\partial y}+\frac{\partial v}{\partial x}\right) \\
& \tau^{x z}=\mu\left(\frac{\partial u}{\partial z}+\frac{\partial w}{\partial x}\right), \quad \tau^{y z}=\mu\left(\frac{\partial v}{\partial z}+\frac{\partial w}{\partial y}\right)
\end{aligned}
$$


The parameter $\mu$ is called the coefficient of dynamic viscosity. For incompressible fluids for which $\rho$ is constant, it is customary to define another constant $\nu=\frac{\mu}{\rho}$ which is called the coefficient of kinematic viscosity. We substitute the expression (2.5) into (2.4). For example in the first equation in (2.4) the viscous terms are

$$
\begin{aligned}
\frac{\partial}{\partial x} & \left(\frac{\partial u}{\partial x}+\frac{\partial u}{\partial x}\right)+\frac{\partial}{\partial y}\left(\frac{\partial u}{\partial y}+\frac{\partial v}{\partial x}\right)+\frac{\partial}{\partial z}\left(\frac{\partial u}{\partial z}+\frac{\partial w}{\partial x}\right) \\
& =\frac{\partial^{2} u}{\partial x^{2}}+\frac{\partial^{2} u}{\partial y^{2}}+\frac{\partial^{2} u}{\partial z^{2}}+\frac{\partial}{\partial x}\left(\frac{\partial u}{\partial x}\right)+\frac{\partial}{\partial x}\left(\frac{\partial v}{\partial y}\right)+\frac{\partial}{\partial x}\left(\frac{\partial w}{\partial z}\right) \\
& =\nabla^{2} u+\frac{\partial}{\partial x}(\nabla \cdot \vec{u})
\end{aligned}
$$

Since the flow is incompressible $\nabla \cdot \vec{u}=0$ and so the viscous term is simply $\nabla^{2} u$. Similarly in the second and the third equations in (2.4) the viscous terms are $\nabla^{2} v$ and $\nabla^{2} w$, respectively.

The momentum equations (2.4) can thus be written as

$$
\begin{gathered}
\left(\frac{D u}{D t}+f_{*} w-f v\right)=-\frac{1}{\rho} \frac{\partial p}{\partial x}+\nu \nabla^{2} u \\
\left(\frac{D v}{D t}+f u\right)=-\frac{1}{\rho} \frac{\partial p}{\partial y}+\nu \nabla^{2} v \\
\left(\frac{D w}{D t}-f_{*} u\right)=-\frac{1}{\rho} \frac{\partial p}{\partial z}-g+\nu \nabla^{2} w
\end{gathered}
$$

Equations (2.6)-(2.8) are the Navier-Stokes equations for an incompressible rotating fluid flow. Geophysical flows are in general nearly inviscid and so one is justified in setting $\nu=0$. The Navier-Stokes equations with $\nu$ set to zero are called the Euler equations. 


\subsubsection{Energy Equation}

The first law of thermodynamics states that the rate of change of total energy is equal to the rate of work done by the system plus the rate of heat addition. In other words, the energy of any particle in the moving fluid does not change, when we consider adiabatic motion of the fluid in which the entropy remains constant. This law can be written as

$$
\rho \frac{D e}{D t}=-p(\nabla \cdot \mathbf{u})+\Phi-\nabla \cdot q
$$

where $e$ is the energy, the first term on the right hand side is the work done due to compression or expansion, $\Phi$ is the work done by friction forces, $q$ is the heat flux per unit area. Equations (2.3), (2.6)-(2.8) and (2.9) completely describe the evolution of the fluid. In general, however, it is complicated to solve these equations analytically unless many simplifying assumptions are made.

For incompressible inviscid flow the energy equation can be simplified. As we mentioned before for an incompressible fluid the continuity equation is $\nabla \cdot \mathbf{u}=0$. For an inviscid flow $\Phi=0$. If the fluid also satisfies the perfect gas law then it can be shown that the energy $e$ is a linear function of temperature $T$. If it is also assumed that the heat flux $q$ satisfies Fourier's law

$$
q=-k \nabla \cdot T
$$


then the energy equation can be written in terms of $T$ in the form

$$
\frac{D T}{D t} \sim \nabla \cdot T
$$

In this simplified problem we can solve for $T$ independently of the other dependent variables $u, v, w$ and $\rho$. To solve for the velocity components we do not need to know $T$. Since our problem deals with incompressible inviscid flow we will only consider (2.3) and (2-6)-(2.8).

\subsection{Streamfunction and vorticity}

For a two-dimensional incompressible fluid with velocity components $u$ and $v$ in the $x y$-plane the continuity equation becomes

$$
\nabla \cdot \mathbf{u}=\frac{\partial u}{\partial x}+\frac{\partial v}{\partial y}=0
$$

and the momentum equations are

$$
\begin{gathered}
\left(\frac{D u}{D t}-f v\right)=-\frac{1}{\rho} \frac{\partial p}{\partial x}+\nu \nabla^{2} u \\
\left(\frac{D v}{D t}+f u\right)=-\frac{1}{\rho} \frac{\partial p}{\partial y}+\nu \nabla^{2} v
\end{gathered}
$$

We can use the continuity equation to define another fluid property called the streamfunction. The streamfunction $\Psi$ serves as a scalar potential for the veloc- 
ity and is defined as

$$
\frac{\partial \Psi}{\partial x}=v, \text { and } \frac{\partial \Psi}{\partial y}=-u
$$

The lines of constant $\Psi$ are everywhere tangent to the velocity field at a given time. Hence there is no flow perpendicular to them. These lines are streamlines.

We also define a fluid property called the vorticity that measures the extent of rotation in the fluid. The vorticity is defined as the curl of the velocity,

$$
\zeta \equiv \nabla \times \mathbf{u}
$$

In a two-dimensional flow, $w=0$ and $\frac{\partial}{\partial z}=0$, so the vorticity is related to the streamfunction by the vorticity defined as the $z$ component of $\nabla \times \mathbf{u}$ so

$$
\zeta=\frac{\partial v}{\partial x}-\frac{\partial u}{\partial y}=\nabla^{2} \psi
$$

The vorticity equation can be derived by manipulating the momentum equation (2.11)-(2.12). We differentiate equation (2.11) by $y$ and (2.12) by $x$. In order to eliminate the pressure gradient from the equations, we subtract the first from the second. Using the relation (2.13) gives the streamfunction-vorticity formulation

$$
\frac{D \zeta}{D t}=(\mathbf{u} \cdot \nabla) \zeta+\nu \nabla^{2} \zeta-\frac{\partial f}{\partial y} \Psi_{x}
$$

Thus in terms of the streamfunction we get

$$
\nabla^{2} \Psi_{t}-\Psi_{x} \nabla^{2} \Psi_{y}+\Psi_{y} \nabla^{2} \Psi_{x}+\frac{\partial f}{\partial y} \Psi_{x}=\nu \nabla^{4} \Psi
$$


where the subscripts denote partial differentiation.

\subsection{The Coriolis force and the $\beta$-plane approxi- mation}

If we consider an object in uniform motion, in a rotating system with the axis of rotation perpendicular to the plane of motion, the resulting path of the object would be a curved line with a direction opposite to the direction of coordinate rotation. The force that defines this curved path is the Coriolis force. In geophysical fluid dynamics, we consider the Earth to be a perfect sphere rotating around its northpole to south-pole axis with rotation rate $\omega$. The angular velocity is given by

$$
\Omega=(0,2 \omega \cos \phi, 2 \omega \sin \phi)=\left(0, f_{*}, f\right)
$$

as a vector along the rotation axis, $\omega$ is the rotation rate of the Earth and $\phi$ is the latitude. And also consider the absolute acceleration minus the centrifugal component $\frac{d \mathbf{u}}{d t}+2 \Omega \times \mathbf{u}$ which has three components

$$
\begin{gathered}
\frac{d u}{d t}+2 \omega \cos \phi w-2 \omega \sin \phi v \\
\frac{d v}{d t}+2 \omega \sin \phi u \\
\frac{d w}{d t}-2 \omega \cos \phi u
\end{gathered}
$$

The term $f=2 \Omega \sin \phi$ is called the Coriolis parameter and $f_{*}=2 \Omega \cos \phi$ is the reciprocal Coriolis parameter. In the northern hemisphere, $f$ is positive, at the 
equator it is zero and it is negative in the southern hemisphere. $f_{*}$ is zero at the poles and positive in both hemispheres. To study wave motion in a horizontal plane we define a local Cartesian frame of reference by eastward and northward coordinates, $x$ and $y$, respectively. We can expand the Coriolis parameter in Taylor series about a fixed latitude $\phi_{0}$ as

$$
f=f_{0}+\beta y+\text { higher order terms, }
$$

where $\beta \equiv\left(\frac{d f}{d y}\right)_{\phi_{0}}$ and $y=0$ at $\phi_{0}$. If $L$ is the latitudinal scale of the motion, then in the expansion of $f$,

$$
\frac{\beta L}{f_{0}} \sin \frac{\cos \phi_{0} L}{\sin \phi_{0} r}
$$

Thus if the latitudinal scale is much smaller than the radius of the Earth, i.e., if $\frac{L}{r} \ll 1$, then $\frac{d f}{d y}=\beta$ can be assumed to be constant. This approximation is called the $\beta$-plane approximation.

Substituting the $\beta$-plane approximation $f \sim f_{0}+\beta y$ into the equation for conservation of potential vorticity and setting the viscosity to zero gives

$$
\nabla^{2} \Psi_{t}-\Psi_{x} \nabla^{2} \Psi_{y}+\Psi_{y} \nabla^{2} \Psi_{x}+\beta \Psi_{x}=0
$$

This is the barotropic vorticity equation and it will be starting point for our analysis. 


\section{Chapter 3}

\section{Geophysical flows}

\subsection{Nondimensionalization}

The starting point for this study is the barotropic vorticity equation (2.17) which was derived in chapter 2 and is in terms of dimensional variables and parameters. Let us represent these dimensional quantities as $x^{*}, y^{*}, t^{*}, \Psi^{*}$ and $\beta^{*}$. The dimensional Laplacian operator is the usual Laplacian operator in two variables $\nabla^{* 2}=\frac{\partial^{2}}{\partial x^{* 2}}+\frac{\partial^{2}}{\partial y^{* 2}}$. We write the equation as

$$
\nabla^{* 2} \Psi_{t^{*}}^{*}+\Psi_{x^{*}}^{*} \nabla^{* 2} \Psi^{*}{ }_{y^{*}}-\Psi^{*}{ }_{y^{*}} \nabla^{* 2} \Psi_{x^{*}}^{*}+\beta^{*} \Psi^{*}{ }_{x^{*}}=0
$$

To allow comparisons between different scales in the problem we non-dimensionalize our equation with respect to typical length scales $L_{x}$ and $L_{y}$ in the zonal and meridional directions respectively and $U$ a typical velocity scale. The non-dimensional 
variables (without asterisks) are defined in terms of the dimensional ones as:

$$
x=\frac{x^{*}}{L_{x}}, \quad y=\frac{y^{*}}{L_{y}}, \quad t=\frac{U t^{*}}{L_{x}}, \quad \Psi=\frac{\Psi^{*}}{L_{y} U}, \quad \beta=\frac{L_{y}^{2} \beta^{*}}{U} .
$$

Substituting into (3.1) gives

$$
\frac{U^{2}}{L_{x} L_{y}} \nabla^{2} \Psi_{t}+\frac{U^{2}}{L_{x} L_{y}} \Psi_{x} \nabla^{2} \Psi_{y}-\frac{U^{2}}{L_{x} L_{y}} \Psi_{y} \nabla^{2} \Psi_{x}+\frac{U^{2} \beta}{L_{x} L_{y}} \Psi_{x}=0
$$

where we define the non-dimensional Laplacian operator $\nabla^{2}$ as

$$
\nabla^{2}=L_{y}^{2} \nabla^{* 2}=\delta \frac{\partial^{2}}{\partial x^{2}}+\frac{\partial^{2}}{\partial y^{2}}
$$

where $\delta=L_{y}^{2} / L_{x}^{2}$ is the square of the aspect ratio. Multiplying by $L_{y} L_{x} / U^{2}$, the evolution of the flow is found to be described by the non-dimensional barotropic vorticity equation

$$
\nabla^{2} \Psi_{t}+\Psi_{x} \nabla^{2} \Psi_{y}-\Psi_{y} \nabla^{2} \Psi_{x}+\beta \Psi_{x}=0
$$

where $\Psi(x, y, t)$ is the total streamfunction and $\beta$ is the non-dimensional gradient of planetary vorticity in the $y$-direction.

In perturbation theory, the total streamfunction is usually written as the sum of the mean part and the perturbation

$$
\Psi(x, y, t)=\bar{\psi}_{0}(y)+\varepsilon \psi(x, y, t)
$$


The corresponding velocity components for the mean flow and for the disturbance are $\bar{u}(y)=-\bar{\psi}_{0 y}$ and $u^{\prime}=-\psi_{y}, v^{\prime}=\psi_{x}$ respectively. The non-dimensional parameter $\varepsilon$ is defined to be $\varepsilon=\varphi /\left(L_{y} U\right)$ where $\varphi$ is the dimensional amplitude of the forcing and $\varepsilon$ therefore gives a measure of the amplitude of the disturbance relative to the magnitude of the basic flow. The equation for the perturbation streamfunction is

$$
\nabla^{2} \psi_{t}+\bar{u}_{0} \nabla^{2} \psi_{x}+\left(\beta-\bar{u}_{0}^{\prime \prime}\right) \psi_{x}+\varepsilon\left(\psi_{x} \nabla^{2} \psi_{y}-\psi_{y} \nabla^{2} \psi_{x}\right)=0
$$

This can be written in terms of the perturbation vorticity $\zeta(x, y, t)=\nabla^{2} \psi(x, y, t)$, as

$$
\zeta_{t}+{\overline{u_{0}}}_{x}+\left(\beta-{\overline{u_{0}}}^{\prime \prime}\right) \psi_{x}+\varepsilon\left(\psi_{x} \zeta_{y}-\psi_{y} \zeta_{x}\right)=0
$$

If $\varepsilon \ll 1$ the equation can be linearized by setting $\varepsilon=0$.

\subsection{Critical layer}

In this section we shall describe what a critical layer is and what happens to the solution near a critical layer. Setting $\varepsilon=0$ in (3.5) gives the linear equation

$$
\left(\frac{\partial}{\partial t}+\bar{u}_{0} \frac{\partial}{\partial x}\right) \nabla^{2} \psi+\left(\beta-\bar{u}_{0}{ }^{\prime \prime}\right) \frac{\partial}{\partial x} \psi=0
$$

If $\psi$ is assumed to be sinusoidal in $x$ and $t$ it can be written in the form

$$
\psi(x, y, t)=\phi(y) e^{i k(x-c t)}+\text { c.c. }
$$


where $k$ is the zonal wavenumber, $c$ is the phase speed, both assumed to be real, and $\phi(y)$ is the complex amplitude of the streamfunction. By adding the complex conjugate we ensure that $\psi$ is real.

By substituting (3.8) into (3.7) we obtain an ordinary differential equation for the amplitude $\phi$ :

$$
\phi^{\prime \prime}+\left(\frac{\beta-\bar{u}_{0}^{\prime \prime}}{\bar{u}_{0}-c}-\delta k^{2}\right) \phi=0
$$

This equation is known as the Rayleigh-Kuo equation. The equation is singular at any point $y=y_{c}$, where $\overline{u_{0}}\left(y_{c}\right)=c$. Using the method of Frobenius to solve (3.9) gives two linearly independent series solutions near the singular point $y=y_{c}$

$$
\phi(y)=a \phi_{a}(y)+b \phi_{b}(y)
$$

where $a$ and $b$ are constants. This solution is not valid at $y=y_{c}$ since at that point the zonal component of the perturbation velocity $u^{\prime}=-\psi_{y}$ has a logarithmic singularity. Near the singular point $y_{c}$ the leading order term of the solution is $\left(y-y_{c}\right) \log \left(y-y_{c}\right)$. Lin (1966) derived this solution and then included viscosity in a critical layer around the singularity and derived a nonsingular critical layer solution. He showed that for negative $\left(y-y_{c}\right)$ the logarithm must be defined as

$$
\log \left(y-y_{c}\right)=\log \left|y-y_{c}\right|+i \theta \operatorname{sgn}\left(\bar{u}_{c}^{\prime}\right)
$$

with $\theta=-\pi$. This means that there is a "phase shift" of $\theta=-\pi$ across the critical layer.

The general solution of (3.9) is $\phi=a^{ \pm} \phi_{a}(y)+b^{ \pm} \phi_{b}(y)$, and the constants are 
found by matching the inviscid outer solution and the viscous critical layer solution

$$
\begin{gathered}
b^{+}=b^{-}=b, \\
a^{+}-a^{-}=i \frac{\left(\beta-\bar{u}_{c}^{\prime \prime}\right)}{\left|\bar{u}_{c}^{\prime}\right|} b \theta .
\end{gathered}
$$

Consequently the streamfunction perturbation is discontinuous across the critical layer and there is a corresponding discontinuity in the wave momentum flux. Of particular interest is the meridional flux of wave momentum flux which is defined as $\bar{\rho} u v$ where $\bar{\rho}$ is the density of basic flow, $u$ and $v$ are the velocity components of the perturbation. In our configuration where $\bar{\rho}$ is constant it is customary to set $\bar{\rho}=1$ and consider the average of $u v$ in the $x$ direction. The average is defined over a zonal wavelength and we shall denote it by an overbar, as follows

$$
F(y, t)=-\frac{k}{2 \pi} \int_{0}^{2 \pi / k} u v \mathrm{~d} x=-\overline{u v}
$$

This averaged momentum flux is called the Reynold's stress. We first note that for waves that are sinusoidal in $x, y$ and $t$ the Reynold's stress is constant. It is straightforward to show this. If we write $\psi=A e^{i(k x+l y-k c t)}+$ c.c. then

$$
u=-i l A e^{i(k x+l y-k c t)}+i l A^{*} e^{-i(k x+l y-k c t)}
$$

and

$$
v=i l A e^{i(k x+l y-k c t)}-i l A^{*} e^{-i(k x+l y-k c t)}
$$


so

$$
F=-u v=-2 k l|A|^{2}
$$

is constant, and if $k$ and $l$ are positive, $F$ is negative.

In the more general problem that we are studying

$$
\psi=\phi(y, t) e^{i k x}+\phi^{*}(y, t) e^{-i k x}
$$

and the Reynold's stress is a function of $y$ and $t$. Since we will be using the streamfunction as the dependent variable it will be convenient to write the momentum flux in terms of the streamfunction. The velocity components are

$$
u=-\Psi_{y}=-\left(\phi_{y} e^{i k x}+\phi_{y}{ }^{*} e^{-i k x}\right)
$$

and

$$
v=\Psi_{x}=i k \phi e^{i k x}-i k \phi^{*} e^{-i k x}
$$

Thus,

$$
-u v=\Psi_{x} \Psi_{y}=i k \phi \phi_{y} e^{2 i k x}-i k \phi^{*} \phi_{y}{ }^{*} e^{-2 i k x}+i k \phi \phi_{y}{ }^{*}-i k \phi^{*} \phi_{y}
$$

Averaging over a wavelength $2 \pi / k$ gives

$$
F=-\overline{u v}=i k \phi \phi_{y}{ }^{*}-i k \phi^{*} \phi_{y}=2 k \operatorname{Im}\left(\phi^{*} \phi_{y}\right)
$$

In the absence of a critical layer $F$ is approximately constant (independent of $y$ ) 
if the wave amplitude is oscillatory in the $y$ direction, i.e., if $\phi \sim e^{i l y}$. If $k$ and $l$ are positive $F$ is negative. In problems with critical layers the momentum flux is discontinuous across the critical layer, $F$ is negative between the forced boundary and the critical layer and the it goes to zero at the critical layer. So there is a jump in $F$ across the critical layer which we shall denote as $[F]$. It can be seen from (3.13) that the phase change is related to the Reynolds stress.

Dickinson (1970) and Warn and Warn (1976) studied the time dependent configuration where $\psi(x, y, t)=\phi(y, t) e^{i k x}+$ c.c and showed that in the limit as $t \rightarrow \infty$ a steady solution is obtained which is equal to the solution of the steady Rayleigh-Kuo equation. So for large time the time dependent solution has similar properties to the steady solution. The waves are absorbed at the critical layer, the momentum flux is negative between the forced boundary and the critical layer, and goes to zero at the critical layer. These conditions were also obtained in the numerical solutions of Béland (1976). In the nonlinear problem $(\varepsilon \neq 0)$ the nonlinear solution behaves like the linear solution at early time, i.e., the critical layer, and as a result there is a change in the basic flow. To understand how the waves affect the basic flow we take the $x$ average of equation (3.4) by integrating each term with respect to $x$ over one wavelength. Let us define $\bar{u}(y, t)=\overline{\Psi_{y}(x, y, t)}$ to be the mean velocity at time $t$. After averaging equation (3.4) and simplifying we obtain

$$
\frac{\partial \bar{u}}{\partial t}=\varepsilon^{2} \frac{\partial(-\overline{u v})}{\partial y}=\varepsilon^{2} \frac{\partial F}{\partial y}
$$

This means that in regions where $F$ is constant the mean flow does not change with time, i.e., $\bar{u}(y, t)=\overline{u_{0}}(y)$ the initial mean velocity. Across the critical layer, there is 
a jump in $F$ and so the mean flow changes with time in the critical layer. Since $F$ is negative above the critical layer and zero below, $[F]$ is positive and so the mean flow is accelerated. 


\section{Chapter 4}

\section{Formulation of the problem for a zonally-varying basic flow}

\subsection{Derivation of the equation}

To study wave propagation we make use of the perturbation method in which each field variable is written as the sum of a basic steady part and a time dependent perturbation that represents the local deviation of the field from the basic part. Thus if $\Psi(x, y, t)$ is the total streamfunction, it can be written as the sum of a contribution from the steady basic flow and a time dependent perturbation which is considered to be a periodic function. The basic flow streamfunction is usually assumed to be a function of $y$ only and it is taken to represent the initial zonal mean value of the total streamfunction $\Psi(x, y, t)$

$$
\Psi(x, y, t)=\bar{\psi}(y)+\varepsilon \psi(x, y, t)
$$




$$
\bar{\psi}_{0}(y)=\overline{\Psi(x, y, 0)}
$$

The basic flow velocity is $\left(\bar{u}_{0}(y), 0\right)$ where $\bar{u}_{0}(y)=-\bar{\psi}_{0 y}$. This is the configuration studied by Stewartson (1978) and Warn and Warn (1978). It is a reasonable approximation for the flow in the stratosphere, i.e., the region in the atmosphere above $50 \mathrm{~km}$. In the stratosphere the basic flow is more or less zonally uniform, i.e., it varies with latitude $y$ but is independent of $x$, the zonal coordinate. However, Enomoto and Matsuda (1999) point out that in troposphere, the lower region of the atmosphere, the zonal variations of the basic flow are generally not negligible and thus a more realistic basic flow in that region would be one that varies in the $x$ direction as well as in $y$. Based on this observation Enomoto and Matsuda carried out a numerical investigation of Rossby wave propagation in the presence of a critical layer in a zonally-varying basic flow. The basic flow was assumed to consist of a zonally-uniform and a zonally-varying component. A different reflection mechanism was found in their results: because of the zonally-varying flow, reflection could take place even in the linear problem where was no wave induced mean flow.

In this thesis we examine a configuration in which the basic flow depends on $x$ as well as $y$, but its variation in the $x$-direction is slow compared with its variation in the $y$-direction. At this point we shall assume that the basic flow has an extra component of the form $\gamma \bar{\psi}_{1}(\mu x, y)$ where $\mu$ and $\gamma$ are nondimensional parameters and both are assumed to be much smaller than unity, so that the total streamfunction is

$$
\Psi(x, y, t)=\bar{\psi}_{0}(y)+\gamma \bar{\psi}_{1}(\mu x, y)+\varepsilon \psi(x, y, t)
$$

It is assumed that $\bar{\psi}_{0}, \bar{\psi}_{1}$ and $\psi$ are $O(1)$ so that, as before, the parameter $\varepsilon$ gives 
a measure of the magnitude of the perturbation relative to that of the basic flow. The parameter $\gamma$ measures the magnitude of the zonally-varying component of the basic flow relative to the zonally-uniform part, while $\mu$ defines the slow variation of the zonally-varying flow with respect to $x$.

To solve for $\psi$ with given $\bar{\psi}_{0}$ and $\bar{\psi}_{1}$, we substitute (4.3) into (3.4). We will use the method of multiple scaling and a new variable $X=\mu x$ to define the slow variation in $x$. We will refer to $X$ as the slow variable and $x$ as the fast variable. We will see that $\psi$ has to depend on $X$ also, so we will write

$$
\Psi(x, X, y, t)=\bar{\psi}_{0}(y)+\gamma \bar{\psi}_{1}(X, y)+\varepsilon \psi(x, X, y, t)
$$

The basic flow velocity has components $\bar{u}_{0}(y)=-\bar{\psi}_{0}^{\prime}(y)$ for the zonally-uniform part and $\bar{u}_{1}(X, y)=-\bar{\psi}_{1 y}$ and $\bar{v}_{1}(X, y)=\mu \gamma \bar{\psi}_{1 X}$ for the zonally-varying part. This means that the $y$ component of the basic flow is non zero but small relative to the $x$ component. The basic flow is weakly non parallel. This is one of the characteristics of boundary layer flow. In fact, our configuration could be generalized and used to study wave propagation in boundary layer flow over a solid boundary. 
Substituting (4.4) into (3.4) gives

$$
\begin{aligned}
& \nabla^{2} \psi_{t}+\bar{u}_{0} \nabla^{2} \psi_{x}+\left(\beta-\bar{u}_{0}^{\prime \prime}\right) \psi_{x} \quad I \\
& +\varepsilon\left(\psi_{x} \nabla^{2} \psi_{y}-\psi_{y} \nabla^{2} \psi_{x}\right) \quad I I \\
& +\mu\left(\bar{u}_{0} \nabla^{2} \psi_{X}+\left(\beta-\bar{u}_{0}^{\prime \prime}\right) \psi_{X}\right) \quad I I I \\
& +\gamma\left(\psi_{x} \nabla^{2} \bar{\psi}_{1 y}-\bar{\psi}_{1 y} \nabla^{2} \psi_{x}\right) \quad I V \\
& +\gamma \mu \varepsilon^{-1}\left(\bar{u}_{0} \nabla^{2} \bar{\psi}_{1 X}+\left(\beta-\bar{u}_{0}^{\prime \prime}\right) \bar{\psi}_{1 X}\right) \quad V \\
& +\gamma \mu\left(\bar{\psi}_{1 X} \nabla^{2} \psi_{y}-\psi_{y} \nabla^{2} \bar{\psi}_{1 X}\right)+\gamma \mu\left(\psi_{X} \nabla^{2} \bar{\psi}_{1 y}-\bar{\psi}_{1 y} \nabla^{2} \psi_{X}\right) \quad V I \\
& +\gamma^{2} \mu \varepsilon^{-1}\left(\bar{\psi}_{1 X} \nabla^{2} \bar{\psi}_{1 y}-\bar{\psi}_{1 y} \nabla^{2} \bar{\psi}_{1 X}\right) \quad V I I \\
& +\varepsilon \mu\left(\psi_{X} \nabla^{2} \psi_{y}-\psi_{y} \nabla^{2} \psi_{X}\right)=0 \quad V I I I
\end{aligned}
$$

The terms are grouped according to the parameter multiplying them and labeled as (I) order 1 terms, (II) $O(\varepsilon)$ terms, (III) $O(\mu)$ terms, (IV) $O(\gamma)$ terms, (V) $O\left(\gamma \mu \varepsilon^{-1}\right)$, (VI) $O(\gamma \mu)$, (VII) $O\left(\gamma^{2} \mu \varepsilon^{-1}\right)$ and (VIII) $O(\varepsilon \mu)$ terms. Depending on the relative magnitude of the three parameters $\varepsilon, \gamma$ and $\mu$ we can obtain a different balance between the terms (II)-(VIII). We have summarized all the different possible scalings for these three parameters in Table 4.1. The Laplacian operator which is defined as (3.3) can be written in terms of $x, X$ and $y$ as

$$
\nabla^{2}=\frac{\partial^{2}}{\partial y^{2}}+\delta \frac{\partial^{2}}{\partial x^{2}}+\delta \mu^{2} \frac{\partial^{2}}{\partial X^{2}}
$$

We will only study the case where $\delta \ll \varepsilon, \delta \ll \mu, \delta \ll \gamma$ and $\delta \ll \gamma \mu \varepsilon^{-1}$ so that we can set $\delta=0$ and omit the $x$ derivative in the Laplacian operator.

If $\delta$ is as large as the other parameters we need to include the $x$ derivative in 
the Laplacian. However at leading order the qualitative behavior of the solution is unchanged by including these $x$ derivatives in the linear problem. Campbell (2004) derived analytic solutions for a configuration with $\delta=0$ and found that the results were in very good agreement with numerical results obtained with larger values of $\delta$. We also rule out the possibility that $(\varepsilon \ll \mu \ll \gamma)$ or $(\varepsilon \ll \gamma \ll \mu)$, since these inequalities both lead to a situation where $\gamma \mu \varepsilon^{-1}>O(1)$ and so the term $\mathrm{V}$ is larger than the term I. The scalings that ensure that $\varepsilon \ll O(1), \mu \ll O(1), \gamma \ll O(1)$ and $\gamma \mu \varepsilon^{-1} \ll O(1)$ are listed in Table 4.1. The leading order terms are shown in the third column.

\begin{tabular}{|l|l|l|c|l|}
\hline case 1 & & $\mu \ll \varepsilon \ll \gamma$ & $\gamma$ & linear \\
\hline case 2 & & $\varepsilon \ll \gamma=\mu$ & $\gamma \mu \varepsilon^{-1}$ & linear \\
\hline case 3 & & $\mu=\varepsilon \ll \gamma$ & $\gamma, \gamma \mu \varepsilon^{-1}$ & linear \\
\hline case 4 & & $\varepsilon=\gamma \ll \mu$ & $\mu, \gamma \mu \varepsilon^{-1}$ & linear \\
\hline case 5 & & $\gamma \ll \varepsilon \ll \mu$ & $\mu$ & linear \\
\hline case 6 & & $\mu \ll \varepsilon=\gamma$ & $\varepsilon, \gamma$ & nonlinear \\
\hline case 7 & (a) & $\mu=\gamma \ll \varepsilon$ & $\varepsilon$ & \\
& (b) & $\mu \ll \gamma \ll \varepsilon$ & $\varepsilon$ & nonlinear \\
& (c) & $\gamma \ll \mu \ll \varepsilon$ & $\varepsilon$ & \\
\hline case 8 & & $\mu=\gamma=\varepsilon$ & $\varepsilon, \gamma, \mu$ & nonlinear \\
\hline case 9 & & $\gamma \ll \mu=\varepsilon$ & $\varepsilon, \mu$ & nonlinear \\
\hline
\end{tabular}

Table 4.1: Parameter scaling

Cases 1-5 give all the possible linear scalings and correspond to situations where the effects of the zonal variation are greater than the effects of nonlinearity. That excludes the case where $\gamma \ll \varepsilon \ll \mu$. For case 5 where $\gamma \ll \varepsilon \ll \mu$ we shall see that 
the zonal variation does not affect the solution. This is described in section 5.2. We shall examine cases 1, 2, 3 and 4 in detail in sections 5.3-5.5 and derive approximate solutions based on the largest terms in the linear equation in each case.

Case 7 gives the nonlinear equation comprising I and II. It corresponds to a situation where the effects of nonlinearity dominate. This is the problem that was studied by Warn and Warn (1978). We shall discuss it in chapter 6. Cases 8, 9 give scalings that include both the nonlinear terms and the terms arising from the zonal variation of the basic flow. In that case the solution would include terms representing both effects.

\subsection{Linear scalings}

We shall consider all 5 linear cases. For the moment the cases of interest are cases 1, 2, 3. Cases 1, 2 and 3 are correspond to: (1) $\mu \ll \varepsilon \ll \gamma,(2) \varepsilon \ll \gamma=\mu$, (3) $\mu=\varepsilon \ll \gamma,(4) \varepsilon=\gamma \ll \mu$. Case 1 corresponds to a case where $\mu=\varepsilon^{p}, \gamma=\varepsilon^{q}$ with $p>1, q>1$. The largest terms in (4.5) are the (I) terms which are $O(1)$, followed by the (III) which are $O(\gamma)$, and all the remaining terms are of $O\left(\gamma^{2}\right)$ or higher. In case 2 we can choose for example, $\varepsilon=\gamma^{p}=\mu^{p} \ll 1$ with $p>1$, so the largest terms in (4.5) are (I) followed by the $O\left(\gamma \mu \varepsilon^{-1}\right)$ (III). Case 3 corresponds to a case where $\mu=\varepsilon=\gamma^{p} \ll 1$ with $p>1$, so that the largest terms are the terms $O(1)$, followed by the $O(\gamma)$ terms (II) and the $O\left(\gamma \mu \varepsilon^{-1}\right)$ terms (V) which are also $O(\gamma)$.

We shall see in section 5.2 that in case 5 the $O(\mu)$ terms are zero so up to that order the solution is comprises just the $O(1)$ part. Case 4 is considered in section 5.5. In each of these balances, some of the terms arising from the zonal variations 
in the basic flow are larger than nonlinear terms. These balances thus allow us the opportunity to examine the effect of the basic flow zonal variation in the context of a linear problem. In chapter 5 we derive solutions for each of these cases. For case 1 after linearization we have the equation

$$
\nabla^{2} \psi_{t}+\bar{u}_{0} \nabla^{2} \psi_{x}+\left(\beta-\bar{u}_{0}^{\prime \prime}\right) \psi_{x}+\gamma\left(\psi_{x} \nabla^{2} \bar{\psi}_{1 y}-\bar{\psi}_{1 y} \nabla^{2} \psi_{x}\right)=0
$$

For case 2 the linear equation is

$$
\nabla^{2} \psi_{t}+\bar{u}_{0} \nabla^{2} \psi_{x}+\left(\beta-\bar{u}_{0}^{\prime \prime}\right) \psi_{x}+\gamma\left(\bar{u}_{0} \nabla^{2} \bar{\psi}_{1 X}+\left(\beta-\bar{u}_{0}^{\prime \prime}\right) \bar{\psi}_{1 X}\right)=0
$$

For case 3 the linear equation is

$$
\begin{gathered}
\nabla^{2} \psi_{t}+\bar{u}_{0} \nabla^{2} \psi_{x}+\left(\beta-\bar{u}_{0}^{\prime \prime}\right) \psi_{x}+\gamma\left(\psi_{x} \nabla^{2} \bar{\psi}_{1 y}-\bar{\psi}_{1 y} \nabla^{2} \psi_{x}\right)+ \\
\gamma\left(\bar{u}_{0} \nabla^{2} \bar{\psi}_{1 X}+\left(\beta-\bar{u}_{0}^{\prime \prime}\right) \bar{\psi}_{1 X}\right)=0
\end{gathered}
$$

We solve each of these linear equations by writing the solution in powers of the parameter $\gamma$

$$
\psi \sim \psi^{(0)}+\gamma \psi^{(1)}+\cdots
$$

Substituting this form into the linear equation in each case gives a series of equations for $\psi^{(0)}, \psi^{(1)}$ and $\ldots$ which are solved successively. In each case, the $O(1)$ equation is

$$
\nabla^{2} \psi_{t}^{(0)}+\psi_{x}^{(0)}\left(\beta-\bar{u}_{0}^{\prime \prime}\right)+\bar{u}_{0} \nabla^{2} \psi_{x}^{(0)}=0
$$

Once this equation is solved we substitute it into the $O(\gamma)$ equation to obtain $\psi^{(1)}$ 
in each case. The $O(1)$ equation is the linear equation that is obtained in the configuration where the basic flow is zonally-uniform $(\varepsilon=\mu=\gamma=0)$. We shall describe its solution in the next section.

\subsection{Solution of the $\mathrm{O}(1)$ equation}

For a zonally-uniform linear Rossby wave in shear flow $\bar{u}_{0}(y), \varepsilon=\mu=\gamma=0$, the solution is $\psi=\psi^{(0)}$, and we only have the $O(1)$ equation

$$
\nabla^{2} \psi_{t}+\bar{u}_{0} \nabla^{2} \psi_{x}+\left(\beta-\bar{u}_{0}^{\prime \prime}\right) \psi_{x}=0
$$

Warn and Warn (1976) solved this equation for the special case in which $\bar{u}_{0}=y$ and

the aspect ratio $\frac{L_{y}}{L_{x}}$ is small enough so that $\delta$ can be set to zero in the Laplacian operator. Campbell and Maslowe (1998) extended Warn and Warn's solution to take into account a forcing in the form of a spatially localized wave packet. In this section we give an overview of the derivation of the solution for our configuration following the procedure used by Campbell and Maslowe (1998).

In general, equation (4.9) can be solved by writing

$$
\psi(x, y, t)=\phi(y, t) e^{i k x}+\text { c.c. }
$$

to give an equation for $\phi(y, t)$ and then taking a Laplace transform in time, and applying the method of Frobenius to find the first few terms in the series solution of the transformed equation. In the special case where $\delta=0$ and $\bar{u}_{0}$ is a linear function of $y$, the transformed equation can be solved exactly and the solution can 
be represented in terms of modified Bessel functions. In the linear problem, the qualitative behavior of the solutions is unchanged by considering this special case (Campbell, 2004). There is no loss in generality in setting $\beta=1$. To do that we will derive the nondimensional variables $y$ and $t$ in terms of the dimensional variables $y^{*}$ and $t^{*}$ by $y=y^{*} /\left(\beta L_{y}\right)$ and $t=\beta U t^{*} / L_{x}$. In summary, the assumptions made in this special case are

$$
\delta=0, \quad \beta=1, \quad \bar{u}_{0}=y
$$

The $O(1)$ equation (4.9) then becomes

$$
\left(\frac{\partial}{\partial t}+y \frac{\partial}{\partial x}\right) \psi_{y y}+\psi_{x}=0
$$

We solve this equation on a semi-infinite rectangular region $-\infty<y \leq y_{1}$ and $0 \leq x \leq 2 \pi$. A sinusoidal boundary condition

$$
\psi\left(x, y_{1}, t\right)=e^{i k x}+\text { c.c. }
$$

is applied at $y=y_{1}$. This is intended to represent the forcing of Rossby waves at northern latitudes and propagating southwards. The boundary condition implies that the solution of the linear problem is of the form

$$
\psi(x, y, t)=\phi(y, t) e^{i k x}+\text { c.c. }
$$

We require that $\psi \rightarrow 0$ as $y \rightarrow-\infty$ and that the solution be bounded for all time. 
Substituting (4.12) into (4.11), gives

$$
\left(\frac{\partial}{\partial t}+i k y\right) \phi_{y y}+i k \phi=0
$$

with boundary condition $\phi\left(y_{1}, t\right)=1$ and initial condition $\phi_{y y}=0$ at $t=0$, i.e., that the vorticity is zero at $t=0$.

To solve (4.13) we take a Laplace transform in $t$, and we obtain

$$
(s+i k y) \tilde{\phi}_{y y}+i k \tilde{\phi}=0
$$

where $\tilde{\phi}(y, s)=\mathcal{L}[\phi(y, t)]$, with boundary condition $\tilde{\phi}\left(y_{1}, s\right)=\frac{1}{s}$. This equation can be transformed into a modified Bessel equation by making the substitution $Y=y-\frac{i s}{k}$ and $w=2 \frac{\tilde{\phi}}{\gamma}$. By letting $z=(-Y)^{1 / 2}$ we find that the solution of (4.14) is

$$
\tilde{\phi}=a z \mathcal{K}_{1}(2 z)+b z \mathcal{I}_{1}(2 z)
$$

where $\mathcal{K}_{1}$ and $\mathcal{I}_{1}$ are modified Bessel functions of order 1 . For the solution to be bounded as $y \rightarrow-\infty$, the constant $b$ must be zero. Applying the boundary condition at $y=y_{1}$ gives

$$
\tilde{\phi}^{(0)}=\frac{1}{s} \frac{\left(\frac{i s}{k}-y\right)^{1 / 2} \mathcal{K}_{1}\left(2\left(\frac{i s}{k}-y\right)^{1 / 2}\right)}{\left(\frac{i s}{k}-y_{1}\right)^{1 / 2} \mathcal{K}_{1}\left(2\left(\frac{i s}{k}-y_{1}\right)^{1 / 2}\right)},
$$

and $\psi^{(0)}$ can be found by taking an inverse Laplace transform. Let us first define

$$
F(s)=\frac{\left(\frac{i s}{k}-y\right)^{1 / 2} \mathcal{K}_{1}\left(2\left(\frac{i s}{k}-y\right)^{1 / 2}\right)}{\left(\frac{i s}{k}-y_{1}\right)^{1 / 2} \mathcal{K}_{1}\left(2\left(\frac{i s}{k}-y_{1}\right)^{1 / 2}\right)}
$$


so that

$$
\phi^{(0)}=\mathcal{L}^{-1}\left[\frac{F(s)}{s}\right]=\frac{1}{2 \pi i} \int_{\alpha-i \infty}^{\alpha+i \infty} \frac{e^{s t}}{s} \frac{\left(\frac{i s}{k}-y\right)^{1 / 2} \mathcal{K}_{1}\left(2\left(\frac{i s}{k}-y\right)^{1 / 2}\right)}{\left(\frac{i s}{k}-y_{1}\right)^{1 / 2} \mathcal{K}_{1}\left(2\left(\frac{i s}{k}-y_{1}\right)^{1 / 2}\right)} \mathrm{d} s
$$

The contour of integration is chosen to lie to the right of all the singularities of the integrand in order to ensure that the integrand is bounded for $t>0$. The integrand has a pole at $s=0$ and two branch points $s=-i k y$ and $s=-i k y_{1}$. The residue at the pole is found to be

$$
\lim _{s \rightarrow 0} s\left[\frac{F(s)}{s}\right]=\frac{(-y)^{1 / 2} \mathcal{K}_{1}\left(2(-y)^{1 / 2}\right)}{\left(-y_{1}\right)^{1 / 2} \mathcal{K}_{1}\left(2\left(-y_{1}\right)^{1 / 2}\right)}
$$

To evaluate the contribution to the integral from the branch points, we integrate around the closed contour shown in Fig A.1. The details are given in Appendix A.

The contribution from the branch point $s=-i k y$ is found to be

$$
I \sim i \pi \frac{e^{-i k y t}}{t^{2} k^{2} y\left(y-y_{1}\right)^{1 / 2} \mathcal{K}_{1}\left(2\left(y-y_{1}\right)^{1 / 2}\right)}
$$

while the contribution from the branch point $s=-i k y_{1}$ is found to be

$$
I^{\prime} \sim-4 i \pi \frac{e^{-i k y_{1} t}}{t^{2} k^{2} y_{1}}\left(y_{1}-y\right)^{1 / 2} \mathcal{K}_{1}\left(2\left(y_{1}-y\right)^{1 / 2}\right)
$$


According to Cauchy's theorem the integral of (4.17) is the sum of

$$
\begin{aligned}
\phi^{(0)} & \sim \frac{(-y)^{1 / 2} \mathcal{K}_{1}\left(2(-y)^{1 / 2}\right)}{\left(-y_{1}\right)^{1 / 2} \mathcal{K}_{1}\left(2\left(-y_{1}\right)^{1 / 2}\right)} \\
& -\frac{e^{-i k y t}}{2 t^{2} k^{2} y\left(y-y_{1}\right)^{1 / 2} \mathcal{K}_{1}\left(2\left(y-y_{1}\right)^{1 / 2}\right)} \\
& +2 \frac{e^{i k y_{1} t}\left(y_{1}-y\right)^{1 / 2} \mathcal{K}_{1}\left(2\left(y_{1}-y\right)^{1 / 2}\right)}{t^{2} y_{1} k^{2}}
\end{aligned}
$$

Thus the solution of equation (4.9) can be written as

$$
\psi^{(0)}=e^{i k x}\left[h_{1}^{(0)}(y)+\frac{e^{-i k y t}}{t^{2}} h_{2}^{(0)}(y)+\frac{e^{-i k y_{1} t}}{t^{2}} h_{3}^{(0)}(y)\right]+\text { c.c. }
$$

where

$$
\begin{gathered}
h_{1}^{(0)}(y)=\frac{(-y)^{1 / 2} \mathcal{K}_{1}\left(2(-y)^{1 / 2}\right)}{\left(-y_{1}\right)^{1 / 2} \mathcal{K}_{1}\left(2\left(-y_{1}\right)^{1 / 2}\right)} \\
h_{2}^{(0)}(y)=-\frac{1}{2 k^{2} y\left(y-y_{1}\right)^{1 / 2} \mathcal{K}_{1}\left(2\left(y-y_{1}\right)^{1 / 2}\right)} \\
h_{3}^{(0)}(y)=\frac{2\left(y_{1}-y\right)^{1 / 2} \mathcal{K}_{1}\left(2\left(y_{1}-y\right)^{1 / 2}\right)}{y_{1} k^{2}}
\end{gathered}
$$

For small $z$ the modified Bessel function $\mathcal{K}_{1}$ can be written as

$$
\mathcal{K}_{1}(z)=\frac{1}{2}\left(\frac{1}{2} z\right)^{-1}+\log \left(\frac{1}{2} z\right) \mathcal{I}_{1}(z)+\cdots
$$

This tells us that $h_{1}^{(0)}(y)$ and $h_{2}^{(0)}(y)$ are singular at $y=0$. As $y \rightarrow 0, h_{1}^{(0)}(y) \sim$ $O(y \log y)$ and $h_{2}^{(0)}(y) \sim O(y)$. Thus, the solution (4.22) breaks down near the critical latitude $(y=0)$. In the limit as $t \rightarrow \infty$ the time dependent terms in (4.22) 
vanish and a steady singular solution is obtained. In the more general problem without the assumptions (4.10), the steady solution obtained in the limit $t \rightarrow \infty$ is the solution (3.10), which behaves like $\left(y-y_{c}\right) \log \left(y-y_{c}\right)$ as $y \rightarrow y_{c}$. Thus the critical layer behavior of the solution is unchanged by having made the assumptions (4.10). Around $y=y_{c}$ a critical layer is defined and the inner solution is found there.

In our problem where $\bar{u}_{0}=y$ the critical layer is defined around $y=0$. 


\section{Chapter 5}

\section{Derivation of the linear solutions}

\subsection{The zonally-varying flow}

In this chapter we derive approximate solutions for the linear equations (4.6), (4.7), (4.8) and also case (4) starting with case 1 . As in section 4.3 we consider the configuration given by the assumptions (4.10). We need to specify the form of the zonally-varying part of the basic flow $\bar{\psi}_{1}(\mu x, y)$.

First let us define the variable $X=\mu x$. Since $\mu \ll 1, X$ varies slowly compared with $x$. We shall refer to $X$ as the slow $x$-variable and $x$ as the fast $x$-variable. We require that the solution $\psi \rightarrow 0$ as $X \rightarrow \pm \infty$ and is bounded as $y \rightarrow-\infty$ so must specify that

$$
\bar{\psi}_{1}(X, y) \rightarrow 0 \text { as } X \rightarrow \pm \infty
$$

and

$$
\bar{\psi}_{1}(X, y) \rightarrow 0 \text { as } y \rightarrow-\infty
$$


Moreover since we wish to compare our results with the conclusions of Enomoto and Matsuda (1999) we choose $\bar{\psi}_{1}(X, y)$ to be a function that can be written as a product of a function of $X$ that goes to zero as $X \rightarrow \pm \infty$ and a function of $y$ that goes to zero as $y \rightarrow-\infty$.

In order to derive explicit expressions for our solutions we shall define $\bar{\psi}_{1}(X, y)=$ $B(y) e^{-X^{2}}$. The solution described in this chapter also could be obtained by specifying the more general form $\bar{\psi}_{1}(X, y)=B(y) A(X)$ provided

$$
A \rightarrow 0 \text { as } X \rightarrow \pm \infty
$$

We shall leave the function $B(y)$ unspecified but restrict it to be nonsingular for all $-\infty<y \leq y_{1}$ to ensure that it does not contribute to the singular critical layer behavior of the solution.

\subsection{The $O(\mu)$ term}

In chapter 4 we derived the solution of the $O(1)$ equation. With the specified boundary condition, the $O(1)$ solution depends on $x, y$ and $t$ but is independent of slow variable $X$. In this section we consider the case 5 in which $\gamma \ll \varepsilon \ll \mu$ and the leading order terms are

$$
\nabla^{2} \psi_{t}+\bar{u}_{0} \nabla^{2} \psi_{x}+\left(\beta-\bar{u}_{0}^{\prime \prime}\right) \psi_{x}+\mu\left(\bar{u}_{0} \nabla^{2} \psi_{X}+\left(\beta-\bar{u}_{0}^{\prime \prime}\right) \psi_{X}\right)=0
$$


We seek the solution in powers of $\mu$,

$$
\psi \sim \psi^{(0)}+\mu \psi^{(1)}+\cdots
$$

and substitute into equation (5.1). At leading order we get an equation for $\psi^{(0)}$ with solution (4.22). At $O(\mu)$ we obtain

$$
\begin{aligned}
& \psi_{y y t}^{(1)}+\bar{u}_{0} \psi_{y y x}^{(1)}+\left(\beta-\bar{u}_{0}^{\prime \prime}\right) \psi_{x}^{(1)} \\
& +\mu\left[\bar{u}_{0} \psi_{y y X}^{(0)}+\left(\beta-\bar{u}_{0}^{\prime \prime}\right) \psi_{X}^{(0)}\right]=0
\end{aligned}
$$

Since $\psi^{(0)}$ is independent of $X$ the $O(\mu)$ term is zero. To solve for $\psi^{(1)}$ substitute

$$
\psi^{(1)}(x, X, y, t)=e^{i k x} \phi^{(1)}(X, y, t)
$$

and then take a Laplace transform of the resulting equation. After applying the boundary condition it turns out that $\psi^{(1)}=0$.

\subsection{Case 1}

In this section we derive an approximate solution for case 1 . The leading order terms in the governing equation in that case are $I$ and $I V$ so the equation is

$$
\nabla^{2} \psi_{t}+\bar{u}_{0} \nabla^{2} \psi_{x}+\left(\beta-\bar{u}_{0}^{\prime \prime}\right) \psi_{x}+\gamma\left(\psi_{x} \nabla^{2} \bar{\psi}_{1 y}-\bar{\psi}_{1 y} \nabla^{2} \psi_{x}\right)=0
$$


We seek a solution in powers of $\gamma$

$$
\psi \sim \psi^{(0)}+\gamma \psi^{(1)}+\cdots
$$

Substitution of this series into (5.4) gives a series of equations for $\psi^{(0)}, \psi^{(1)}, \ldots$ which are solved successively. At $O(1)$ the solution for $\psi^{(0)}$ is the same as equation $(4.22)$.

At $O(\gamma)$ the equation for $\psi^{(1)}$ is non-homogeneous with the non-homogeneous term being dependent on the known function $\bar{\psi}_{1}(X, y)=B(y) e^{-X^{2}}$ (the basic flow) and on the $O(1)$ solution $\psi^{(0)}$ which is given in equation (4.22). The equation for $\psi^{(1)}$ is

$$
\nabla^{2} \psi_{t}^{(1)}+\bar{u}_{0} \nabla^{2} \psi_{x}^{(1)}+\left(\beta-\bar{u}_{0}^{\prime \prime}\right) \psi_{x}^{(1)}=-\left(\psi_{x}^{(0)} \nabla^{2} \bar{\psi}_{1 y}-\bar{\psi}_{1 y} \nabla^{2} \psi_{x}^{(0)}\right)
$$

As before, we make the assumptions (4.10). At this order, the disturbance stream function is written in the form

$$
\psi^{(1)}=\phi^{(1)}(X, y, t) e^{i k x}+\text { c.c. }
$$

and we obtain the following equation for the amplitude $\phi^{(1)}$ :

$$
\left(\frac{\partial}{\partial t}+i k y\right) \phi_{y y}^{(1)}+\phi^{(1)}=i k e^{-X^{2}} N(y, t)
$$

where

$$
N(y, t)=B^{\prime}(y) \phi_{y y}^{(0)}(y, t)-B^{\prime \prime \prime}(y) \phi^{(0)}(y, t)
$$


To solve (5.7) we take the Laplace transform with respect to $t$. The result is

$$
\left(y-\frac{i s}{k}\right) \tilde{\phi}_{y y}^{(1)}+\tilde{\phi}^{(1)}=i k e^{-X^{2}} \tilde{N}(y, s)
$$

where $\tilde{\phi}^{(1)}(y, s)=\mathcal{L}\left[\phi^{(1)}(y, t)\right]$ and $\tilde{N}(y, s)=\mathcal{L}[N(y, t)]$.

We then use the method of variation of parameters to solve (5.9). The details of the solution procedure are given in Appendix B. The solution $\tilde{\phi}^{(1)}(y, s)$ is the sum of the homogeneous equation and a particular solution of the non-homogeneous equation.

Up to this point we have not specified the function $B(y)$. However the solution can not be simplified any further without specifying the function $B(y)$. Since $B(y)$ is required to be a smooth function with no singularity its choice does not affect the qualitative behavior of the inner solution. Since $B$ is nonsingular near $y=0, B(y)$ can be approximated by the first few terms in the Taylor series as $y \rightarrow 0$.

$$
B(y) \sim b_{0}+b_{1} y+O\left(y^{2}\right)
$$

Thus for small $y$, the leading order term in the function $N(y, t)$ is $b_{1} \phi_{y y}^{(0)}$. In Appendix B.1 we make this approximation to simplify the solution for $\psi^{(1)}$. In that case the solution takes the form

$$
\psi^{(1)} \sim e^{i k x} h_{1}^{(1)}(y)+O\left(\frac{1}{t}\right)
$$

where

$$
h_{1}^{(1)}=b_{1} e^{-X^{2}}\left[\frac{G\left(2\left(-y_{1}\right)^{1 / 2}\right) H\left(2(-y)^{1 / 2}\right)}{H\left(2\left(-y_{1}\right)^{1 / 2}\right)}+\frac{G\left(2(-y)^{1 / 2}\right)}{H\left(2(-y)^{1 / 2}\right)}\right]
$$

and $G(z)$ is the function given in (B.17) in Appendix B.1 and $H(z)=\frac{z}{2} \mathcal{K}_{1}(z)$. As 
$y \rightarrow 0$ the first term is $O(y \log y)$.

\subsection{Case 2}

In this section we consider case 2 , i.e., the configuration in which $\varepsilon=\gamma \ll \mu \ll 1$. The governing equation in this case is

$$
\nabla^{2} \psi_{t}+\bar{u}_{0} \nabla^{2} \psi_{x}+\left(\beta-\bar{u}_{0}^{\prime \prime}\right) \psi_{x}+\alpha\left(\bar{u}_{0} \nabla^{2} \bar{\psi}_{1 X}+\left(\beta-\bar{u}_{0}^{\prime \prime}\right) \bar{\psi}_{1 X}\right)=0
$$

We shall define a new parameter $\alpha=\gamma \mu \varepsilon^{-1}$. We shall see that the presence of the $O(\alpha)$ term gives a term in the solution that grows linearly with time. As before, we make the assumptions (4.10). Substituting the function form of $\bar{\psi}_{1}(X, y)$ into (5.11) gives

$$
\psi_{y y t}+y \psi_{y y x}+\psi_{x}-\alpha 2 X e^{-X^{2}}\left(y B^{\prime \prime}(y)+B(y)\right)=0
$$

Let us combine all the non-homogeneous terms and denote them

$$
M(X, y)=+2 X e^{-X^{2}}\left(y B^{\prime \prime}(y)+B(y)\right)
$$

As before, we seek a solution of the form

$$
\psi \sim \psi^{(0)}+\alpha \psi^{(1)}+\cdots
$$


and substitute this into (5.12). At $O(1)$ the solution is the same as in chapter 4, i.e.,

$$
\psi^{(0)} \sim e^{i k x}\left[h_{1}^{(0)}(y)+\frac{e^{-i k y t}}{t^{2}} h_{2}^{(0)}(y)+\frac{e^{-i k y_{1} t}}{t^{2}} h_{3}^{(0)}(y)\right]
$$

At $O(\alpha)$, we get the following equation

$$
\psi_{y y t}^{(1)}+y \psi_{y y x}^{(1)}+\psi_{x}^{(1)}-M(X, y)=0
$$

and we can solve this non-homogeneous equation to find $\psi^{(1)}$. Note that $\psi^{(1)}$ is independent of $\psi^{(0)}$ in this case. The solution of (5.14) is the sum of a particular solution of the non-homogeneous equation and the solution $\psi^{(1)}$ of the corresponding homogeneous equation.

The homogeneous equation is the same as the $O(1)$ equation. Since the nonhomogeneous term $M(X, y)$ is independent of $x$, the particular solution of the nonhomogeneous equation will also be $x$ independent. The solution of the homogeneous equation, on the other hand, depends on $x$. To solve it we write $\psi^{(1)}$ in the form $\phi^{(1)}(X, y, t) e^{i k x}$ and then take the Laplace transform and obtain an equation for $\tilde{\phi}^{(1)}(X, y, s)$ the Laplace transform of $\phi^{(1)}(X, y, t)$. The solution of the transformed equation that is bounded as $y \rightarrow-\infty$ is the solution of the form

$$
a(x, X, s)\left(y-\frac{i s}{k}\right)^{1 / 2} \mathcal{K}_{1}\left(2\left(y-\frac{i s}{k}\right)^{1 / 2}\right) .
$$

This is the $x$ dependent part of the solution and it must satisfy a zero boundary condition at $y=y_{1}$. Thus $a(x, X, s)=0$ and so $\psi_{h}^{(1)}=0$.

Since the non-homogeneous term in (5.14) does not depend on the fast $x$ - 
scale, a particular solution of the non-homogeneous equation must take the form $\psi_{p}^{(1)}(X, y, t)$, independent of $x$. Thus the non-homogeneous equation is

$$
\psi^{(1)}{ }_{y y t}=M(X, y)
$$

Equation (5.15) can be solved by direct integration. Integrating with respect to $t$ and using the initial condition of zero vorticity gives

$$
\phi_{y y}=M(X, y) t
$$

This is then integrated twice with respect to $y$ to give

$$
\phi=M_{1}(X, y) t+y a_{1}(X, t)+a_{2}(X, t)
$$

where $M_{1 y y}=M(X, y)$. The requirement that $\phi \rightarrow 0$ as $y \rightarrow-\infty$ tells us that $a_{1}(X, t)=0=a_{2}(X, t)$. Thus at $O(\alpha)$

$$
\phi=M_{1}(X, y) t
$$

and therefore the solution grows with time. At $t \sim O\left(\alpha^{-1}\right)$ it becomes the same order of magnitude as the $O(1)$ term. The solution takes the form

$$
\psi=e^{i k x} O(1)+\alpha O(t)+\cdots
$$

This tells us that for $t \gg O\left(\alpha^{-1}\right)$ we need to introduce a new scale for the late time 
evolution of the solution. We define a slow time variable $T=\alpha t$. We consider the solution (5.16) to the early time solution and we seek a late time solution. In terms of the new time scale we have

$$
\frac{\partial}{\partial t} \rightarrow \alpha \frac{\partial}{\partial T}
$$

The early time solution (5.17) can be written in terms of the late time variable as

$$
\psi(x, X, y, T) \sim e^{i k x} h_{1}^{(0)}(y)+M_{1}(X, y) T+O(\alpha)
$$

In order to match with the early time solution, the late time solution must take the form

$$
G(x, X, y, T)=G^{(0)}(x, X, y, T)+\alpha G^{(1)}(x, X, y, T)+\cdots
$$

So the late time solution does not depend on $t$. We substitute (5.19) into (5.12) to give

$$
\alpha G_{y y T}^{(0)}+y G_{y y x}^{(0)}+G_{x}^{(0)}+\alpha y G_{y y x}^{(1)}+\alpha G_{x}^{(1)}=\alpha M(X, y)
$$

At $O(1)$ we obtain

$$
y G_{x y y}{ }^{(0)}+G_{x}{ }^{(0)}=0
$$

The general form of the solution of (5.21) is

$$
G^{(0)}(x, X, y, T)=e^{i k x} g_{1}(X, y, T)+g_{2}(X, y, T)
$$

To match with early time solution as $T \rightarrow 1$ we must also have

$$
g_{2}^{(0)} \rightarrow 0 \text { as } T \rightarrow 1
$$


Thus the $O(1)$ solution is

$$
G^{(0)}(X, x, y, T) \rightarrow e^{i k x} h_{1}^{(0)}(y)
$$

At $O(\alpha)$ the equation becomes

$$
G_{y y T}^{(0)}+y G_{y y x}^{(1)}+G_{x}^{(1)}=M(X, y)
$$

In order to avoid getting secular terms, i.e., terms proportional to $x$ we specify that

$$
y G_{y y x}^{(1)}+G_{x}^{(1)}=0
$$

and

$$
G_{y y T}^{(0)}=M(X, y)
$$

Equation (5.23) implies that

$$
g_{2 y y T}^{(0)}=M(X, y)
$$

which given us

$$
g_{2 y y}^{(0)}=M(X, y) T
$$

which matches with the early time solution. The $x$ independent part continues to grow with $T$. To find a bounded solution we would need to include more terms in our solution either the nonlinear $O(\varepsilon)$ terms or the $O(\mu)$ terms. In the next section we consider the effect of adding the $O(\mu)$ terms to our equation. This gives case 4 describe in section 5.6 . 


\subsection{Late time solution}

We consider case $4(\varepsilon=\gamma \ll \mu)$ which means that $\alpha=\mu \gamma \varepsilon^{-1}=\mu$ so the leading order terms are $O(1), O(\mu), O(\alpha)$. After making the assumptions (4.10) and defining $M(X, y)=-2 X e^{-X^{2}}\left(y B^{\prime \prime}(y)+B(y)\right)$ as before we obtain

$$
\psi_{y y t}+y \psi_{y y x}+\psi_{x}+\alpha\left(y \psi_{y y X}+\psi_{X}\right)-\alpha M(X, y)=0
$$

We introduce the late time variable $T=\mu t=\alpha t$. The governing equation in terms of late time variable is

$$
\alpha \psi_{y y T}+y \psi_{y y x}+\psi_{x}+\alpha\left(y \psi_{y y X}+\psi_{X}\right)-\alpha M(X, y)=0
$$

As in case 2 the late time solution does not depend on $t$. We seek a late time solution in powers of $\alpha$.

$$
\psi(x, X, y, T)=G^{(0)}(x, X, y, T)+\alpha G^{(1)}(x, X, y, T)
$$

The $O(1)$ equation is the same as in case 2, equation (5.20). The solution of the $O(1)$ equation satisfies the boundary condition at $y=y_{1}$ is of the form

$$
G^{(0)}(x, X, y, T)=e^{i k x} h_{1}^{(0)}(y)+g_{2}(X, y, T)
$$

In order to match (5.27) with the early time solution as $T \rightarrow 1$, we should have $g_{2}(X, y, 1)=0$. 
At $O(\alpha)$ we have

$$
\begin{aligned}
& \left.y G_{y y x}^{(1)}+G_{x}^{(1)}=-\left[G_{y y T}^{(0)}+y G_{y y X}^{(0)}+G_{X}^{(0)}\right)-M(X, y)\right] \\
& \quad=-\left[g_{2 y y T}+y g_{2 y y X}+g_{2 X}-M(X, y)\right]
\end{aligned}
$$

The right hand side of (5.28) is independent of $x$, so taking the integral with respect to $x$ of both sides gives

$$
y G_{y y}^{(1)}+G^{(1)}=-x\left[g_{2 y y T}+y g_{2 y y X}+g_{2 X}-M(X, y)\right]
$$

As $x \rightarrow \pm \infty$ the solution would be unbounded. So we should have

$$
g_{2 y y T}+y g_{2 y y X}+g_{2 X}=M(X, y)
$$

which is a nonhomogeneous equation for $g_{2}$. Thus the solution of (5.30) is the sum of the solution of the corresponding homogeneous equation which we shall call $g^{H}$ and a particular solution $g^{p}$. The homogeneous equation

$$
g_{2 y y T}^{H}+y g_{2 y y X}^{H}+g_{2 X}^{H}=0
$$

can be solved by defining the transformation $\xi=\frac{X}{T}$. The transformed equation is

$$
\frac{-X}{T^{2}} g_{2 y y \xi}^{H}+\frac{y}{T} g_{2 y y \xi}^{H}+\frac{1}{T} g_{2 \xi}^{H}=0
$$


which simplifies (5.32) to give

$$
(y-\xi) g_{2 y y \xi}^{H}+g_{2 \xi}^{H}=0
$$

The solutions of (5.33) are modified Bessel functions. Therefore

$$
g^{H}{ }_{\xi}=a(\xi)(y-\xi)^{1 / 2} \mathcal{K}_{1}\left(2(y-\xi)^{1 / 2}\right)+b(\xi)(y-\xi)^{1 / 2} \mathcal{I}_{1}\left(2(y-\xi)^{1 / 2}\right)
$$

where $a(\xi)$ and $b(\xi)$ can be determined from the boundary condition and matching condition. We integrate with respect to $\xi$ to find $g^{H}$ and get

$$
g_{H}=\int a(\xi)(y-\xi)^{1 / 2} \mathcal{K}_{1}\left(2(y-\xi)^{1 / 2}\right) \mathrm{d} \xi+f(y)
$$

As $X \rightarrow \pm \infty$, which is equivalent to $\xi \rightarrow \pm \infty$ for finite $t$, we have $g^{H}=0$ and therefore $f(y)=0$.

To find a particular solution of (5.30), we first note that the right hand side of the equation is independent of $T$, so we can find a particular solution that is independent of $T$ by solving the equation

$$
y g_{2 y y X}+g_{2 X}=-2 X e^{-X^{2}}\left(y B^{\prime \prime}(y)+B(y)\right)
$$

We integrate (5.36) with respect to $X$ to get

$$
y g_{2 y y}+g_{2}=e^{-X^{2}}\left(y B^{\prime \prime}(y)+B(y)\right)
$$


which could be solved using variation of parameters. The solutions of the form $g^{p}=e^{-X^{2}} f_{p}(y)$ where $f_{p}(y)$ a linear combination of the modified Bessel functions $\mathcal{I}_{1}$ and $\mathcal{K}_{1}$. Thus the general solution for the equation (5.30) is

$$
g_{2}(X, y, T)=g_{H}\left(\frac{X}{T}, y\right)+g_{p}(X, y)
$$

However this solution can not match the early time solution and also satisfy the boundary condition at $y=y_{1}$. From this we conclude that we need to consider extra terms in our late time solution, for example the $O(\varepsilon)$ terms.

\subsection{Case 3}

In this section, we consider the case $3(\mu=\varepsilon \ll \gamma)$ which means that $\gamma=\gamma \mu \varepsilon^{-1}$, i.e., $\alpha=\gamma$. In fact, case 3 is the combination of case 1 and case 2 since the leading order terms are $O(1), O(\gamma)$ and $O\left(\gamma \mu \varepsilon^{-1}\right)$. So we can find the solution for case 3 by making use of the solutions for case 1 and 2 derived in sections 5.3 and 5.4. In case 3 , the $O(1)$ and $O(\gamma)$ terms are

$$
\begin{aligned}
& \nabla^{2} \psi_{t}+\bar{u}_{0} \nabla^{2} \psi_{x}+\left(\beta-\bar{u}_{0}^{\prime \prime}\right) \psi_{x}+\gamma\left(\psi_{x} \nabla^{2} \bar{\psi}_{1 y}-\bar{\psi}_{1 y} \nabla^{2} \psi_{x}\right) \\
& +\gamma\left(\bar{u}_{0} \nabla^{2} \bar{\psi}_{1 X}+\left(\beta-\bar{u}_{0}^{\prime \prime}\right) \bar{\psi}_{1 X}\right)=0
\end{aligned}
$$

As before we let $\bar{\psi}_{1}(X, y)=B(y) e^{-X^{2}}$, make the assumptions (4.10), and substitute

$$
\psi \sim \psi^{(0)}+\gamma \psi^{(1)}+\cdots
$$


The $O(1)$ equation is the same as in the previous sections, i.e.,

$$
\psi^{(0)}=e^{i k x}\left[h_{1}^{(0)}(y)+\frac{e^{-i k y t}}{t^{2}} h_{2}^{(0)}(y)+\frac{e^{-i k y_{1} t}}{t^{2}} h_{3}^{(0)}(y)\right]
$$

However, at $O(\gamma)$ there are extra terms containing products of $\bar{\psi}_{0}$ and $\bar{\psi}_{1}$ and their derivatives. Since $\bar{\psi}_{0}$ and $\bar{\psi}_{1}$ are the components of the basic flow, they are not periodic. In fact, both functions depend on the slow variable $X$, as well as on $y$, but not on the fast variable $x$. Thus, the result of the extra terms is that the $O(\gamma)$ solution includes an extra term that does not depend on the fast $x$ scale. The simplified governing equation for case 3 is

$$
\begin{aligned}
& \psi_{y y t}+y \psi_{y y x}+\psi_{x}+\gamma\left(-\psi_{x} B^{\prime \prime \prime}(y) e^{-X^{2}}-B^{\prime}(y) e^{-X^{2}} \psi_{y y x}\right) \\
& +\gamma\left(-2 X e^{-X^{2}}\left(y B^{\prime \prime}(y)+B(y)\right)\right)=0
\end{aligned}
$$

As in case 2, we let $M(X, y)=+2 X e^{-X^{2}}\left(y B^{\prime \prime}(y)+B(y)\right)$ and substitute this into (5.40). At $O(1)$ the solution is the same as in chapter 4. At $O(\gamma)$, we solve a non-homogeneous equation to determine $\psi^{(1)}$ as the sum of the solution of the homogeneous equation and particular solution of the non-homogeneous equation. The equation is

$$
\begin{aligned}
& \psi_{y y t}^{(1)}+y \psi_{y y x}^{(1)}+\psi_{x}^{(1)} \\
& \quad+\left[-\psi_{x}^{(0)} B^{\prime \prime \prime}(y) e^{-X^{2}}-B^{\prime}(y) e^{-X^{2}} \psi_{y y x}^{(0)}\right]-M(X, y)=0
\end{aligned}
$$

The general solution of the homogeneous equation is the same as in case 1 . The 
solution of non-homogeneous equation comprises two parts; one which depends on the fast scale $x$ corresponding to term IV and the other corresponding to term $\mathrm{V}$, which is independent of $x$. Thus, the solution can be written as

$$
\psi^{(1)}(X, x, y, t)=e^{i k x} \phi^{(1)}(X, y, t)+\bar{\phi}(X, y, t)
$$

The term $\bar{\phi}(X, y, t)$ depends only on the slow scale $X$ but not on the fast scale $x$. It is a "long wave" centered about the zero wave number. Substitution of the form (5.42) into equation (5.41) gives

$$
\begin{aligned}
& e^{i k x}\left[\left(\frac{\partial}{\partial t}+i k y\right) \phi_{y y}^{(1)}+i k \phi^{(1)}\right]+\frac{\partial}{\partial t} \bar{\phi}_{y y}= \\
& \quad e^{i k x}\left[i k \phi^{(0)} B^{\prime \prime \prime}(y) e^{-X^{2}}+B^{\prime}(y) e^{-X^{2}} \phi_{y y}^{(0)}\right]+M(X, y)
\end{aligned}
$$

We thus obtain two equations at $O(\gamma)$ :

$$
\begin{aligned}
& e^{i k x}\left[\left(\frac{\partial}{\partial t}+i k y\right) \phi_{y y}^{(1)}+i k \phi^{(1)}\right]= \\
& \quad e^{i k x}\left[i k \phi^{(0)} B^{\prime \prime \prime}(y) e^{-X^{2}}+B^{\prime}(y) e^{-X^{2} \phi_{y y}^{(0)}}\right]
\end{aligned}
$$

for the component of the solution centered at wave number $k$ and

$$
\bar{\phi}_{y y t}=M(X, y)
$$

So as in case 2 we have a term centered at wave number 0 which grows with time. Equation (5.44) is the equation solved in case 1. The solution satisfying the boundary and initial conditions can be found in Appendix B. 
Thus the solution in case 3 is

$$
\psi=e^{i k x} O(1)+\gamma e^{i k x} O(1)+\gamma M_{1}(X, y) O(t)
$$

As discussed in section 5.4, the last term grows with time, and when $t>O\left(\gamma^{-1}\right)$ it becomes larger than $O(1)$. To find a solution for this late time regime we can define a late time variable $T=\gamma t$ which is $O(1)$ when $t \sim O\left(\gamma^{-1}\right)$.

\subsection{The critical layer thickness}

In the linear problem with a zonally uniform basic flow, the critical layer thickness is $y \sim O\left(t^{-1}\right)$ (Warn and Warn 1976). In our linear problem with the zonallyvarying basic flow the same critical layer thickness applies since the leading order term is the same. In their study, (Warn and Warn 1976) considered the three regions $|y| t \gg 1,|y| t \sim O(1)$ and $|y| t \ll 1$. These three regions are represented in the Fig 5.1. They showed that the nonlinear terms are the same order of magnitude as the linear terms when $t>O\left(\varepsilon^{-1 / 2}\right)$. In that time regime, the critical layer thickness is $|y| \sim O\left(t^{-1 / 2}\right) \sim O\left(\varepsilon^{1 / 2}\right)$ as shown in Fig 5.1.

In our linear problem the same critical layer thickness $|y| \sim O\left(t^{-1}\right)$ applies since the leading order term is the same as in the case without the zonal variation of the basic flow. But our solutions in cases 2 and 3 show that at $t \sim O\left(\alpha^{-1}\right)$ the term resulting from the zonal variation is the same order of magnitude as the leading order term. Our late time regime starts at $t \sim O\left(\alpha^{-1}\right)$. This is shown in Fig 5.1. 


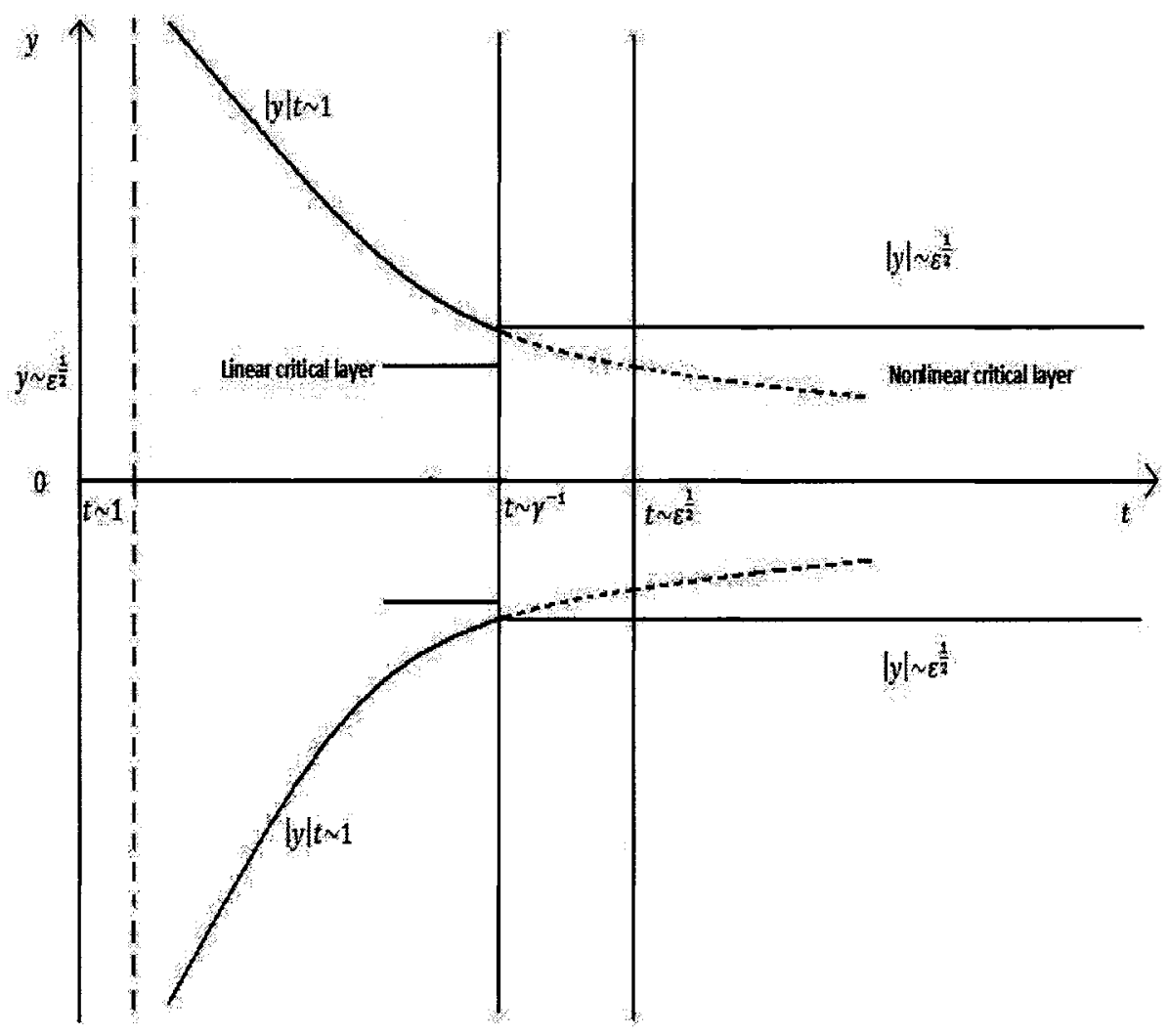

Figure 5.1: Time regime comparison 


\subsection{Inner solution}

The functions $h_{1}^{(0)}(y)$ and $h_{1}^{(1)}(y)$ in the $O(1)$ solution that were derived in section 5.1 are singular as $y \rightarrow 0$, so that the outer solution is not valid there. We need to define a critical layer around the singular point $y=0$.

For $|y| \ll 1$ we can not approximate the integrand in (4.17) by the first term in the series expansion for $\rho$, since the first of the neglected terms would be $O(1)$ after integrating. We need to retain all the terms in the expansion for $(-\rho-i k y)$. The procedure for evaluating the integrand in this regime is described in Appendix C. The solution turns out to be

$$
\begin{aligned}
& \psi(x, y, t)=e^{i k x}\left\{h_{1}^{(0)}(y)+\frac{h_{2}^{(0)}(y)}{k t} E_{2}^{\left(\operatorname{sgn}_{y}\right)}(i k|y| t)\right. \\
& \left.+h_{4}(y) E_{3}^{(\operatorname{Sgn} y)}(i k|y| t)+e^{-i k y_{1} t}\left[O\left(t^{-1}\right)+O\left(t^{-2}\right)\right]\right\}
\end{aligned}
$$

where

$$
\begin{aligned}
& E_{k}^{+}(z)=\int_{1}^{\infty} \frac{e^{-z u}}{u^{k}} \mathrm{~d} u, \quad \operatorname{Re} \mathrm{z} \geq 0 \\
& E_{k}^{-}(z)=\int_{1}^{\infty} \frac{e^{+z u}}{u^{k}} \mathrm{~d} u, \quad \operatorname{Re} \mathrm{z} \leq 0 .
\end{aligned}
$$

with $k=0,1,2, \cdots$ and $h_{4}(y)=\frac{1}{4}\left(g_{1}(y)+g_{2}(y)\right)$. Expanding in powers of $y$ gives

$$
(-y)^{1 / 2} \mathcal{K}_{1}\left(2(-y)^{1 / 2}\right) \sim 1-y \log y+(1-2 \lambda) y+\cdots
$$

where Euler's constant, which is usually denoted as $\gamma$, has been denoted as $\lambda$ here to avoid confusion with our parameter $\gamma$. 
At late time $t \sim O\left(\gamma^{-1}\right)$ if $|y| t \sim O(1)$ then $|y| \sim O(\gamma)$. Thus in the critical layer we can define a critical layer variable $Y=\gamma^{-1} y$. The critical layer solution can thus be written as

$$
\psi(x, X, Y, T)=a_{1} e^{i k x} e^{-X^{2}}\left\{1-\gamma Y \log \gamma+\gamma(1-2 \lambda) Y-\gamma Y \log Y+O\left(\gamma^{2}\right)\right\}
$$

with the constant $a_{1}$ given by

$$
a_{1}=\frac{1}{2\left(-y_{1}\right)^{1 / 2} \mathcal{K}_{1}\left(2\left(-y_{1}\right)^{1 / 2}\right)}
$$

and for $Y<0$, the logarithm is defined to be

$$
\log Y=\log |Y|+i \pi
$$

This tells us the form of the critical layer solution. Campbell and Maslowe (1998) considered this critical layer solution and derived a series of inner equations which can be solved to give

$$
\begin{aligned}
\psi \sim a_{1} e^{i k x} e^{-X^{2}} & \left(1-Y \gamma \log \gamma-(\operatorname{sgn} Y)-\gamma\left[\frac{i}{k T}+\frac{\left.i k Y^{2} T\right)}{2}+\frac{k^{2}|Y|^{3} T^{2}}{12}+\cdots\right]+\gamma Y\left(\log k T-\alpha-\frac{i \pi}{2}\right)\right)
\end{aligned}
$$

Campbell and Maslowe (1998) derived the inner equations and matched with the outer solution. We can do the same here. At $O(\gamma)$ our outer solution in case 1 takes 
the form

$$
\psi^{(1)} \sim e^{i k x} h_{1}^{(1)}(y)+O\left(\frac{1}{t}\right)
$$

As $y \rightarrow 0 h_{1}^{(1)}(y) \sim y \log y$ so there are additional terms in our inner solution, which take the form $(\gamma \log \gamma) Y$ and $\gamma Y \log Y$. This tells us that the $O(\gamma)$ term gives a contribution to the singular behavior of the solution in the critical layer.

In case 3 the outer solution is

$$
\psi \sim \psi^{(0)}+\gamma\left[e^{i k x} h_{1}^{(1)}(y)+O\left(\frac{1}{t}\right)\right]+\gamma O(t)
$$

The terms that grow with time are nonsingular so they do not affect the inner solution. Table 5.1 summarizes the solutions in each of the different space and time regimes.

\begin{tabular}{|c|c|c|}
\hline & case 4 & case 4 \\
\hline case 1 & cases 2 and 3 & \\
\hline Outer solution & Outer solution early time & Outer solution late time \\
\hline$A$ & $A+B(t)$ & $A+C\left(\frac{X}{T}\right)$ \\
$A$ is singular as $y \rightarrow 0$ & $A$ is singular as $y \rightarrow 0$ & $A$ is singular as $y \rightarrow 0$ \\
& $B(t) \rightarrow \infty$ as $t \rightarrow \infty$ & C bounded as $T \rightarrow \infty$ \\
\hline inner solution & inner solution early time & inner solution late time \\
\hline$A^{\prime}$ & $A^{\prime}+B(t)$ & $A^{\prime}+C\left(\frac{X}{T}\right)$ \\
\hline
\end{tabular}

Table 5.1: Total view for the late time and early time solution. 


\section{Chapter 6}

\section{Conclusions}

In this thesis we used analytical methods to study Rossby wave propagation in the presence of a critical layer on a $\beta$-plane in a configuration where the basic flow has a zonally-varying component. We considered different scalings of the parameteres $\mu, \varepsilon$ and $\gamma$ that led to linear problems describing small amplitude waves. With the scaling for cases 2 and 3 we found that the solution becomes unbounded for large time.

For zonally-varying uniform basic flow the Reynold's stress, which is defined in (3.11), is discontinuous at the critical layer, and the waves are absorbed there. The Reynold's stress is given by

$$
F=2 k \operatorname{Im}\left(\phi^{*} \phi_{y}\right)=2 k \operatorname{Im}\left(h_{1}^{(0) *} h_{y}^{(0)}\right)
$$

as $t \rightarrow \infty$.

In the nonlinear problem (Warn and Warn, 1978, Béland, 1976) the mean flow 
is accelerated by the waves according to (3.14). Since $F$ is negative above the critical layer, and zero below the critical layer the jump $[F]$ is positive and so the mean velocity $\bar{u}$ is accelerated. This corresponds to absorption of the waves by the mean flow. The change in the mean flow is the zero wavenumber component of the disturbance. In addition, higher wave number components develop corresponding to wavenumbers $2 k, 3 k, 4 k$ and so on. Warn and Warn (1978) concluded that at late time these components become the same order of magnitude as the component corresponding to wavenumber $k$. In the nonlinear problem total momentum flux is the sum over the wavenumbers

$$
F=\sum_{k} F_{k}=\sum_{k} 2 k\left(\operatorname{Im} \phi_{k}^{*} \phi_{k y}\right)
$$

where $\phi_{k}(y, t)$ is the component of the solution corresponding to a wavenumber $k$. Warn and Warn concluded from their solution that at late time the jump $\left[F_{k}\right]$ would have to be zero for all wavenumbers $k$, and so $[F]=0$. Numerical solutions (Béland, 1976) show that at late time $t>O\left(\varepsilon^{-1 / 2}\right),[F]$ goes to zero, then becomes negative and oscillates between negative and positive values. When it is negative the mean flow is accelerated and the waves are reflected.

In our linear problem the momentum flux is

$$
F=2 k \operatorname{Im}\left[h^{*} h_{y}+\left(M_{1} h_{y}+M_{1 y} h^{*}\right) T\right]
$$

The momentum flux does not reach a steady state as $t \rightarrow \infty$ and so the jump $[F]$ could be negative, zero or positive depending on the choice of $B(y)$, which determines 
$M(X, y)$.

Hence we could have reflection or absorption in our linear problem. So our configuration describes a general mechanism which allows for both possibilities (reflection and absorption) in a linear framework. This is in contrast to the case with zonally uniform flow where only absorption can occur in the linear problem. This is also, in general, consistent with Enomoto and Matsuda's (1999) conclusions. The obvious next step in our study would be to consider the scalings that lead to nonlinear problems, i.e., cases 6, 7, 8 and 9 in Table 4.1. With both nonlinear effects and effects of the zonally-varying flow we could expect either absorption or reflection but could also study the interactions between the waves, the basic flow and the higher wavenumbers. 


\section{Appendix A}

\section{Evaluation of the contributions}

\section{from the branch points in case 1}

The $O(1)$ solution was derived by Campbell and Maslowe (1998). In this appendix we describe the procedure used to evaluate the integral (4.17).

To evaluate the integral (4.17) we integrate along the closed contour shown in Fig A.1. The large semicircle has radius $R$ and the small circles have radii $r$, and we take the limit as $R \rightarrow \infty$ and $r \rightarrow 0$ so that the straight part of the semicircle becomes infinite.

Along the curved contour $C_{0}$ we set

$$
s=R e^{i \theta}+s_{0} \quad \Rightarrow d s=i R e^{i \theta} d \theta
$$

then we have

$$
I_{0}=\int_{\pi / 2}^{3 \pi / 2} i R \frac{e^{t\left[R e^{i \theta}+s_{0}\right]}}{R e^{i \theta}+s_{0}} F(\theta) \mathrm{d} \theta
$$


By substituting $e^{i \theta}=\cos \theta+i \sin \theta$, on the contour $C_{0}, \pi / 2<\theta<3 \pi / 2$ and so $-1<\cos \theta<0$ and in the integrand we see that $\lim _{R \rightarrow \infty} e^{t R \cos \theta}=0$. This means that $\lim _{R \rightarrow \infty} I_{0}=0$. Along the contours $C_{3}$ and $C_{3}^{\prime}$, we set

$$
s=r e^{i \theta}+s_{1} \quad \Rightarrow d s=i r e^{i \theta} d \theta
$$

and in each case the integral becomes

$$
I_{3}=\int_{0}^{2 \pi} i r \frac{e^{t\left[r e^{i \theta}+s_{1}\right]}}{r e^{i \theta}+s_{1}} F(\theta) \mathrm{d} \theta
$$

and as $r \rightarrow 0$ the integrand vanishes. Thus the contributions from the contours $C_{3}$ and $C_{3}^{\prime}$ are zero. The only non zero contributions to the integrand come from the straight contours $C_{1}, C_{2}, C_{1}^{\prime}$ and $C_{2}^{\prime}$. Along $C_{2}$ we define a new variable $s=\rho e^{i \pi}-i k y$ so $\frac{i s}{k}-y=-\frac{i \rho}{k}$ and $d s=-d \rho$ and denote the integral along $C_{1}$ by $I_{1}$. Along $C_{1}$,

$$
\left(\frac{i s}{k}-y\right)^{1 / 2}=\left(i e^{i \pi} \frac{\rho}{k}\right)^{1 / 2}=e^{3 i \pi / 4}\left(\frac{\rho}{k}\right)^{1 / 2}
$$

and so the integrand can be written as

$$
I_{1}=\int_{0}^{-\infty} \frac{e^{t(-\rho-i k y)}}{(-\rho-i k y)} \frac{e^{3 i \pi / 4}(\rho / k)^{1 / 2} \mathcal{K}_{1}\left(2 e^{3 i \pi / 4}(\rho / k)^{1 / 2}\right)}{\left(-i \rho / k+y-y_{1}\right)^{1 / 2} \mathcal{K}_{1}\left(2\left(-i \rho / k+y-y_{1}\right)^{1 / 2}\right)} \mathrm{d} \rho
$$

For large $t$, most of the contribution to the integrand comes from values of $\rho$ closes to zero. Thus $I_{1}$ can be approximated by taking the limit of small $\rho$. To leading 
orders in $\rho$, the integrand can be written as

$$
I_{1} \sim \int_{0}^{+\infty} \frac{e^{-\rho t} e^{-i k y t}}{-i k y} \frac{\left(\frac{\rho}{k}\right)^{1 / 2} e^{3 i \pi / 4} \mathcal{K}_{1}\left(2 e^{3 i \pi / 4}\left(\frac{\rho}{k}\right)^{1 / 2}\right)}{\left(y-y_{1}\right)^{1 / 2} \mathcal{K}_{1}\left(2\left(y-y_{1}\right)^{1 / 2}\right)} \mathrm{d} \rho
$$

Along $C_{2}$ define $s=\rho e^{-i \pi}-i k y$, and so $d s=-d \rho$. For large $t$, most of the contribution to the integrand comes from values of $\rho$ closes to zero. Thus $I_{2}$ can be approximated by taking the limit of small $\rho$. To leading orders in $\rho$, the integrand can be written as

$$
I_{2} \sim-\int_{0}^{+\infty} \frac{e^{-\rho t} e^{-i k y t}}{-i k y} \frac{\left(\frac{\rho}{k}\right)^{1 / 2}\left(-e^{3 i \pi / 4}\right) \mathcal{K}_{1}\left(2\left(-e^{3 i \pi / 4}\right)\left(\frac{\rho}{k}\right)^{1 / 2}\right)}{\left(y-y_{1}\right)^{1 / 2} \mathcal{K}_{1}\left(2\left(y-y_{1}\right)^{1 / 2}\right)} \mathrm{d} \rho
$$

The contribution from the branch point $s=-i k y$ is $I=I_{1}+I_{2}$. Making use of the relation $\mathcal{K}_{1}(-z)+\mathcal{K}_{1}(z)=-i \pi \mathcal{I}_{1}(z)$

$I \sim \int_{0}^{+\infty}-\left[\frac{e^{-i k y t} e^{3 i \pi / 4}\left(\frac{\rho}{k}\right)^{1 / 2} e^{-\rho t}}{(-\rho-i k y)\left(-\frac{i \rho}{k}+y-y_{1}\right)^{1 / 2} \mathcal{K}_{1}\left(2\left(-\frac{i \rho}{k}+y-y_{1}\right)^{1 / 2}\right)}\right]\left(i \pi \mathcal{I}_{1}\left(2 e^{3 i \pi / 4}\left(\frac{\rho}{k}\right)^{1 / 2}\right)\right) \mathrm{d} \rho$

finally we find that

$$
I \sim \int_{0}^{+\infty}-\left[\frac{e^{-i k y t} e^{3 i \pi / 4}\left(\frac{\rho}{k}\right)^{1 / 2} e^{-\rho t}}{-i k y\left(y-y_{1}\right)^{1 / 2} \mathcal{K}_{1}\left(2\left(y-y_{1}\right)^{1 / 2}\right)}\right]\left(i \pi \mathcal{I}_{1}\left(2 e^{3 i \pi / 4}\left(\frac{\rho}{k}\right)^{1 / 2}\right)\right) \mathrm{d} \rho
$$

The leading order term of integrand (A.5) is obtained by approximating $\mathcal{I}_{1}(z) \sim z / 2$ and taking the limit as $\rho \rightarrow 0$. This gives

$$
I \sim 2 \pi i \frac{e^{-i k y t}}{t^{2} k^{2} y\left(y-y_{1}\right)^{1 / 2} \mathcal{K}_{1}\left(2\left(y-y_{1}\right)^{1 / 2}\right)}
$$


By a similar procedure along $C^{\prime}{ }_{1}$ for the other branch point, $s=-i k y_{1}$, we find that

$$
I_{1}^{\prime}=-\int_{0}^{+\infty} \frac{-e^{-\rho t} e^{-i k y_{1} t}}{\left(-\rho-i k y_{1}\right)} \frac{\left(-i \frac{\rho}{k}+y_{1}-y\right)^{1 / 2} \mathcal{K}_{1}\left(2\left(-\frac{\rho}{k}+y_{1}-y\right)^{1 / 2}\right)}{\left(-i \frac{\rho}{k}\right)^{1 / 2} e^{3 i \pi / 4} \mathcal{K}_{1}\left(2 e^{3 i \pi / 4}\left(\frac{\rho}{k}\right)^{1 / 2}\right)} \mathrm{d} \rho
$$

and along $\mathrm{C}_{2}^{\prime}$ for,

$$
I_{2}^{\prime}=\int_{0}^{+\infty} \frac{e^{-\rho t} e^{-i k y_{1} t}}{\left(-\rho-i k y_{1}\right)} \frac{\left(-i \frac{\rho}{k}+y_{1}-y\right)^{1 / 2} \mathcal{K}_{1}\left(2\left(-\frac{\rho}{k}+y_{1}-y\right)^{1 / 2}\right)}{-\left(-i \frac{\rho}{k}\right)^{1 / 2} e^{3 i \pi / 4} \mathcal{K}_{1}\left(-2 e^{3 i \pi / 4}\left(\frac{\rho}{k}\right)^{1 / 2}\right)} \mathrm{d} \rho
$$

Again for large $t$, most of the contribution to these integrals comes from values of $\rho$ close to zero and so each integral can be approximated by taking the limit as $\rho \rightarrow 0$. The sum of the two integrals is

$$
\begin{aligned}
I^{\prime}= & I_{1}^{\prime}+I_{2}^{\prime}=\int_{0}^{+\infty} \frac{e^{-\rho t} e^{-i k y_{1} t}}{\left(-\rho-i k y_{1}\right)}\left(-i \frac{\rho}{k}+y_{1}-y\right)^{1 / 2} \\
& \left.\mathcal{K}_{1}\left(2\left(-\frac{\rho}{k}+y_{1}-y\right)^{1 / 2}\right)\right)\left[4 \pi\left(\frac{\rho}{k}\right) \mathcal{I}_{1}\left(2 e^{3 i \pi / 4}\left(\frac{\rho}{k}\right)^{1 / 2}\right)\right] \mathrm{d} \rho
\end{aligned}
$$

and so the contribution from the branch point $s=-i k y_{1}$ is

$$
I^{\prime}=I_{1}^{\prime}+I_{2}^{\prime} \sim-4 \pi i \frac{e^{-i k y_{1} t}}{t^{2} k^{2} y_{1}}\left(y_{1}-y\right)^{1 / 2} \mathcal{K}_{1}\left(2\left(y_{1}-y\right)^{1 / 2}\right)
$$

Therefore according to Cauchy's theorem the integral of (4.17) is the sum of the contribution from the pole and the contribution from branch points along the closed contours $C_{1}, C_{2}, C^{\prime}{ }_{1}$ and $C^{\prime}{ }_{2}$. The integral can be approximated as 


$$
\begin{aligned}
& \phi^{(0)} \sim \frac{(-y)^{1 / 2} \mathcal{K}_{1}\left(2(-y)^{1 / 2}\right)}{\left(-y_{1}\right)^{1 / 2} \mathcal{K}_{1}\left(2\left(-y_{1}\right)^{1 / 2}\right)} \\
& -\frac{e^{-i k y t}}{2 t^{2} k^{2} y\left(y-y_{1}\right)^{1 / 2} \mathcal{K}_{1}\left(2\left(y-y_{1}\right)^{1 / 2}\right)} \\
& +2 \frac{e^{i k y_{1} t}\left(y-y_{1}\right)^{1 / 2} \mathcal{K}_{1}\left(2\left(y-y_{1}\right)^{1 / 2}\right)}{t^{2} y_{1} k^{2}}
\end{aligned}
$$




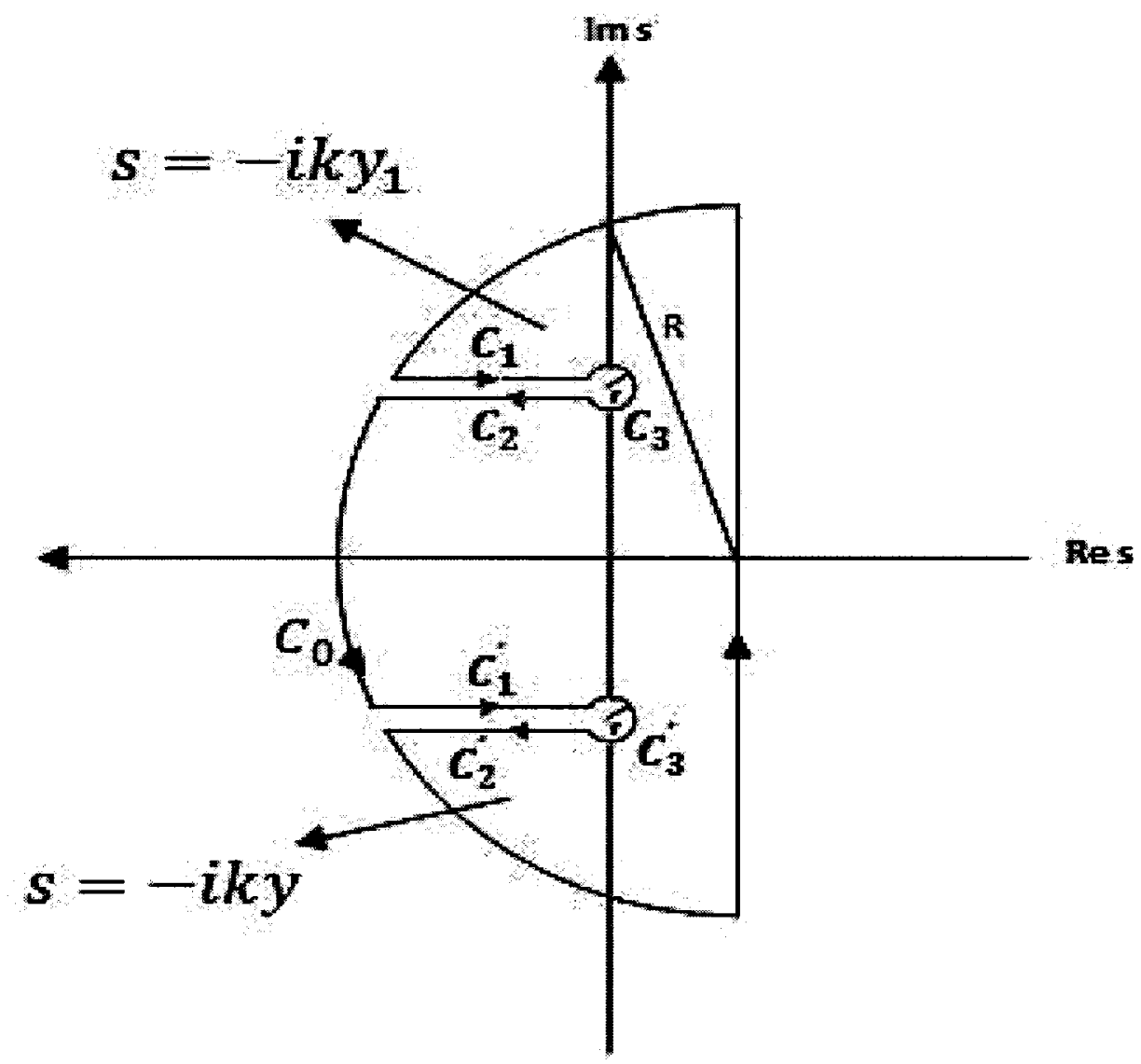

Figure A.1: Contour of integration in the complex $s$-plane for equation (4.17). The branch points $s=-i k y_{1}$ and $s=-i k y$ are indicated by the black dots. 


\section{Appendix B}

\section{Solution of the non-homogeneous}

\section{equation in case 1}

In this section we describe the procedure used for solving the non-homogeneous equation (5.6). For the $O(\gamma)$ term in case 1 , the equation is

$$
\left(\frac{\partial}{\partial t}+i k y\right) \phi_{y y}^{(1)}+i k \phi^{(1)}=i k e^{-X^{2}} N(y, t)
$$

where $N(y, t)=B^{\prime}(y) \phi_{y y}^{(0)}(y, t)-B^{\prime \prime \prime}(y) \phi^{(0)}(y, t)$ and $\phi^{(0)}(y, t)$ is the $O(1)$ solution (4.22). The boundary conditions are $\phi^{(0)}\left(X, y_{1}, t\right)=0$ and $\phi^{(1)}(x, y, t) \rightarrow 0$ as $y \rightarrow-\infty$.

To solve this problem we take the Laplace transform of (B.1) with respect to $t$. The result is

$$
\left(y-\frac{i s}{k}\right) \tilde{\phi}_{y y}^{(1)}+\tilde{\phi}^{(1)}=e^{-X^{2}} \tilde{N}(y, s)
$$

where $\tilde{\phi}^{(0)}(y, s)=\mathcal{L}\left[\phi^{(0)}(y, t)\right]$ and $\tilde{N}(y, s)=\mathcal{L}[N(y, t)]=\left[B^{\prime}(y) \tilde{\phi}_{y y}^{(0)}(y, s)-B^{\prime \prime \prime}(y) \tilde{\phi}^{(0)}(y, s)\right]$. 
Since $\tilde{\phi}^{(0)}$ satisfies

$$
\left(y-\frac{i s}{k}\right) \tilde{\phi}_{y y}^{(0)}+\tilde{\phi}^{(0)}=0
$$

$\tilde{N}(y, s)$ can be written as

$$
\tilde{N}(y, s)=-\left[B^{\prime}(y)+B^{\prime \prime \prime}(y)\left(y-\frac{i s}{k}\right)\right] \frac{\tilde{\phi}^{(0)}}{\left(y-\frac{i s}{k}\right)} .
$$

The solution of the non-homogeneous equation (B.1) is

$$
\tilde{\phi}^{(1)}(X, y, s)=\tilde{\phi}_{h}(X, y, s)+\tilde{\phi}_{p}(X, y, s)
$$

where $\tilde{\phi}_{h}$ is the solution of the corresponding homogeneuos equation and $\tilde{\phi}_{p}$ is a particular solution of (B.1).

The particular solution is obtained using the method of variation of parameters. We write the particular solution as

$$
\tilde{\phi}_{p}(X, y, s)=e^{-X^{2}}\left[u_{1} \tilde{\varphi}_{1}+u_{2} \tilde{\varphi}_{2}\right]
$$

where $\tilde{\varphi}_{1}(y, s)$ and $\tilde{\varphi}_{2}(y, s)$ are linearly independent solutions of the homogeneous equation and $u_{1}$ and $u_{2}$ are given by

$$
\begin{gathered}
u_{1}=-\int \frac{\tilde{\varphi}_{2} N}{W} \\
u_{2}=\int \frac{\tilde{\varphi}_{1} N}{W}
\end{gathered}
$$

with $W$ being the Wronskian of $\tilde{\varphi}_{1}$ and $\tilde{\varphi}_{2}$. The solutions of the homogeneous 
equation are written in terms of modified Bessel functions as

$$
\begin{aligned}
& \tilde{\varphi}_{1}=\left(\frac{i s}{k}-y\right)^{1 / 2} \mathcal{I}_{1}\left(2\left(\frac{i s}{k}-y\right)^{1 / 2}\right), \\
& \tilde{\varphi}_{2}=\left(\frac{i s}{k}-y\right)^{1 / 2} \mathcal{K}_{1}\left(2\left(\frac{i s}{k}-y\right)^{1 / 2}\right) .
\end{aligned}
$$

According to the properties of modified Bessel functions the Wronskian is $W=1$. The $O(1)$ solution $\tilde{\phi}^{(0)}$ can be written as

$$
\tilde{\phi}^{(0)}=\frac{1}{s} \frac{H\left(2\left(\frac{i s}{k}-y\right)^{1 / 2}\right)}{H\left(2\left(\frac{i s}{k}-y_{1}\right)^{1 / 2}\right)}
$$

where $H$ is defined as

$$
H(z)=\frac{z}{2} \mathcal{K}_{1}(z)
$$

and $z=2\left(\frac{i s}{k}-y\right)^{1 / 2}$. The function $\tilde{N}(y, s)$ can also be written in terms of $H$ as

$$
\tilde{N}(y, s)=-\frac{\left[B^{\prime}(y)+B^{\prime \prime \prime}(y)\left(y-\frac{i s}{k}\right)\right]}{s\left(\frac{i s}{k}-y\right)} \frac{H\left(2\left(\frac{i s}{k}-y\right)^{1 / 2}\right)}{H\left(2\left(\frac{i s}{k}-y_{1}\right)^{1 / 2}\right)}
$$

Setting $z=2\left(\frac{i s}{k}-y\right)^{1 / 2}$ and $z_{1}=2\left(\frac{i s}{k}-y_{1}\right)^{1 / 2}, \tilde{N}$ can be written as

$$
\tilde{N}=-\frac{B^{\prime}(y)+B^{\prime \prime \prime}(y) \frac{z^{2}}{4}}{s\left(\frac{-z^{2}}{4}\right)} \frac{H(z)}{H\left(z_{1}\right)}
$$

The functions $u_{1}$ and $u_{2}$ can then be found by substituting (B.6) and (B.7) into (B.4) and (B.5) and once $u_{1}$ and $u_{2}$ are known $\tilde{\phi}^{(1)}$ can be found from (B.3). Since $B(y)$ is not given we can not simplify the solution any further at this stage. In the 
specific case where $B^{\prime \prime \prime}(y)=0$ and $B^{\prime}(y)=b_{1}$ is a constant, we can simplify the expression for $\tilde{\phi}^{(1)}$ and derive expressions for the leading order terms in $\tilde{\phi}^{(1)}$.

\section{B.1 The case $B^{\prime}(y)=\mathbf{a}$ constant}

The function $B(y)$ has not been specified up until now. In fact, as long as $B(y)$ nonsingular at $y=0$, it does not contribute to the singular behavior of the solution. In this section we discuss the special case where $B^{\prime}(y)=b_{1}$ is a constant, and $B^{\prime \prime \prime}=0$ which allows us to simplify our solutions further. This simplification can be considered near the critical layer where $y$ is small. Since $B(y)$ is analytic at $y=0$ it can be expanded as a Taylor series about the point $y=0$ and for small $y$ it can be approximated by the first few terms. The case considered here, corresponds to approximating by the first two terms. Then $\tilde{N}$ can be written as

$$
\tilde{N}=\frac{b_{1}}{s \frac{z^{2}}{4}} \frac{H(z)}{H\left(z_{1}\right)}
$$

where $H(z)=\frac{z}{2} \mathcal{K}_{1}(z), z=2\left(\frac{i s}{k}-y\right)^{1 / 2}$ and $z_{1}=2\left(\frac{i s}{k}-y_{1}\right)^{1 / 2}$. In that case, $u_{1}$ and $u_{2}$ can be obtained by making the change of variables $z=\left(\frac{i s}{k}-y\right)$ in the integrals (B.4) and (B.5). We then find that

$$
\tilde{\phi}_{p}^{(1)}=e^{-X^{2}} \frac{b_{1}}{2 s z_{1} \mathcal{K}_{1}\left(z_{1}\right)}\left[-z \mathcal{I}_{1}(z) \int z \mathcal{K}_{1}{ }^{2}(z) \mathrm{d} z \int z \mathcal{I}_{1}(z) \mathcal{K}_{1}(z) \mathrm{d} z\right]
$$

For small $z$ we can approximate these integrals using approximations for $\mathcal{I}_{1}$ and $\mathcal{K}_{1}$,

$$
\mathcal{I}_{1}(z) \sim \frac{z}{2}
$$


and

$$
\mathcal{K}_{1}(z) \sim \frac{1}{z}+\frac{z}{2} \ln \left(\frac{z}{2}\right)+O\left(z^{2} \ln z\right)+O(z)
$$

So the integrand is

$$
z \mathcal{K}_{1}^{2}(z) \sim \frac{1}{z}+z \ln \left(\frac{z}{2}\right)+\frac{z^{3}}{4}(\ln z)^{2}
$$

which is $O(\log z)$, and

$$
z \mathcal{I}_{1}(z) \mathcal{K}_{1}(z) \sim z+z^{2} \ln \left(\frac{z}{2}\right)
$$

which is $O(z)$.

Thus

$$
\tilde{\phi}_{p} \sim \frac{b_{1}}{2 s z_{1} \mathcal{K}_{1}\left(z_{1}\right)} G(z)
$$

where $G(z) \sim O(z)$ as $z \rightarrow 0$.

The solution of the homogeneous equation that satisfies the boundary condition as $y \rightarrow-\infty$ is the one involving the modified Bessel function of the second kind $\mathcal{K}_{1}$. Thus

$$
\tilde{\phi}_{h}(y, s)=H\left(2\left(\frac{i s}{k}-y\right)^{1 / 2}\right) e^{-X^{2}}
$$

where $H(z)=\frac{z}{2} \mathcal{K}_{1}(z)$. So the solution of the non-homogeneous equation is

$$
\tilde{\phi}^{(1)}(X, y, s)=e^{-X^{2}}\left[A(s) H\left(2\left(\frac{i s}{k}-y\right)^{1 / 2}\right)+\tilde{\phi}_{p}(y, s)\right]
$$

Applying the zero boundary condition at $y=y_{1}$ gives

$$
\tilde{\phi}^{(1)}(X, y, s)=e^{-X^{2}}\left[-\frac{\tilde{\phi}_{p}(y, s)}{H\left(2\left(\frac{i s}{k}-y_{1}\right)^{1 / 2}\right)} H\left(2\left(\frac{i s}{k}-y\right)^{1 / 2}\right)+\tilde{\phi}_{p}(y, s)\right]
$$


where

$$
\tilde{\phi}_{p}(y, s) \sim \frac{b_{1}}{s H\left(2\left(\frac{i s}{k}-y_{1}\right)^{1 / 2}\right)} G\left(\frac{i s}{k}-y\right)
$$

This can be written in terms of $H$ as

$$
\tilde{\phi}^{(1)}(X, y, s)=e^{-X^{2}}\left[-\frac{b_{1} G\left(2\left(\frac{i s}{k}-y\right)^{1 / 2}\right)}{s H\left(2\left(\frac{i s}{k}-y_{1}\right)^{1 / 2}\right)} \frac{H\left(2\left(\frac{i s}{k}-y\right)^{1 / 2}\right)}{H\left(2\left(\frac{i s}{k}-y_{1}\right)^{1 / 2}\right)}+\frac{b_{1} G\left(2\left(\frac{i s}{k}-y\right)^{1 / 2}\right)}{s H\left(2\left(\frac{i s}{k}-y\right)^{1 / 2}\right)}\right]
$$

We invert the Laplace transform to find $\phi^{(1)}(X, y, t)$ by evaluating it in the complex plane along an infinite line lying to the right of all singularities of the integrand.

$$
\phi^{(1)}=\frac{1}{2 \pi i} \int_{\alpha-i \infty}^{\alpha+i \infty} \tilde{\phi}^{(1)}(X, y, s) e^{s t} \mathrm{~d} s
$$

Again there are three singularities, a pole at $s=0$ and branch points $s=-i k y$ and $s=-i k y_{1}$.

The residue at the pole gives us the steady part of the $O(\gamma)$ solution:

$$
\lim _{s \rightarrow 0} s \phi^{(1)} e^{s t}=b_{1} e^{-X^{2}}\left[\frac{G\left(2\left(-y_{1}\right)^{1 / 2}\right) H\left(2(-y)^{1 / 2}\right)}{H\left(2\left(-y_{1}\right)^{1 / 2}\right)}+\frac{G\left(2(-y)^{1 / 2}\right)}{H\left(2(-y)^{1 / 2}\right)}\right]
$$

where

$$
\begin{aligned}
& G(z)=-\frac{z}{2} \mathcal{I}_{1}(z) \times \\
& \quad \int \frac{z}{2} \mathcal{K}_{1}(z) z \mathcal{K}_{1}(z)\left(\frac{-z}{2}\right) \mathrm{d} z+\frac{z}{2} \mathcal{K}_{1}(z) \int \frac{z}{2} \mathcal{I}_{1}(z) z \mathcal{K}_{1}(z)\left(\frac{-z}{2}\right) \mathrm{d} z
\end{aligned}
$$

As $y \rightarrow 0$ the first term in (B.16) is $O(1)+O(y \log y)$ and the second term is $O(y)$. So the leading order part of the solution is the first term. To evaluate the contributions 
from branch points, we use the contour shown in Fig.1. The contributions from the branch points are $O\left(\frac{1}{t}\right)$ so as $t \rightarrow \infty$ the solution becomes (B.16). 


\title{
Appendix C
}

\section{Evaluation of the solution for}

\author{
$|y| t \sim O(1)$
}

In this section we describe the evaluation of the integrals (A.4) and (A.7) near critical layer $y=0$ where $|y| t \sim O(1)$ and $t \gg 1$. The solution obtained in chapter 5 and Appendix A is valid in the outer region where $|y| t \gg O(1)$ and $t \gg 1$. To obtain the integrals $I_{1}$ and $I_{2}$ in (A.2) and (A.3) we approximated the integrand in (A.1) by the first term in its series expansion by taking the limit as $\rho \rightarrow 0$. However, to evaluate the integral (4.17) in the regime where $|y| t \sim O(1)$ we cannot neglect all the terms in the expansion. We need to take into account the fact that $y \ll 1$ as well. To expand (A.4) in powers of $\rho$ there are three functions that should be expanded in their Taylor series around $\rho=0$. The first one is:

$$
\frac{1}{-\rho-i k y}=-\sum_{n=0}^{\infty}\left(\frac{-i \rho}{k y}\right)^{n}
$$


The second one is

$$
(\rho / k)^{1 / 2} \mathcal{I}_{1}\left(2 e^{3 i \pi / 4}(\rho / k)^{1 / 2}\right) \sim e^{3 i \pi / 4}(\rho / k) \sum_{n=0}^{\infty} \frac{(\rho / k)^{n}}{n !(n+1) !}
$$

The third function to expand is

$$
G(\rho, y)=\frac{1}{\left(-i \rho / k+y-y_{1}\right)^{1 / 2} \mathcal{K}_{1}\left(2\left(-i \rho / k+y-y_{1}\right)^{1 / 2}\right)}
$$

use the Bessel function properties $\mathcal{K}_{1}^{\prime}(z)=-\mathcal{K}_{0}(z)+\frac{\mathcal{K}_{0}^{\prime}(z)}{z}$ and $\mathcal{K}_{0}^{\prime}(z)=-\mathcal{K}_{1}(z)$, to expand $G(\rho, y)$ in its Taylor series around $\rho=0$. We get:

$$
G(\rho, y)=G(0, y)+\rho G_{\rho}(0, y)+(1 / 2) \rho^{2} G_{\rho \rho}(0, y)+\cdots
$$

To simplify set $g_{1}(y)=G(0, y)=\frac{1}{\left(y-y_{1}\right)^{1 / 2} \mathcal{K}_{1}\left(2\left(y-y_{1}\right)^{1 / 2}\right)}$. Differentiating $G$ with respect to $\rho$,

$$
\begin{aligned}
G_{\rho}= & \frac{(-i / k)\left(-i \rho / k+y-y_{1}\right)^{-1 / 2} \mathcal{K}_{1}\left(2\left(-i \rho / k+y-y_{1}\right)^{-1 / 2}\right)}{2\left(-i \rho / k+y-y_{1}\right)\left[\mathcal{K}_{1}\left(2\left(-i \rho / k+y-y_{1}\right)^{-1 / 2}\right)\right]^{2}} \\
& -\frac{\left.(-i / k)\left(-i \rho / k+y-y_{1}\right) \mathcal{K}_{1}^{\prime}\left(2\left(-i \rho / k+y-y_{1}\right)^{-1 / 2}\right)\right]}{\left(-i \rho / k+y-y_{1}\right)\left[\mathcal{K}_{1}\left(2\left(-i \rho / k+y-y_{1}\right)^{-1 / 2}\right)\right]^{2}}
\end{aligned}
$$

After setting $\rho=0$ and multiplying $G_{\rho}$ by $\rho$, the result is

$$
\rho G_{\rho}(0, y)=-i \rho / k \frac{\left[\left(y-y_{1}\right)^{-1 / 2} \mathcal{K}_{1}\left(2\left(y-y_{1}\right)^{1 / 2}\right)+\mathcal{K}_{0}\left(2\left(y-y_{1}\right)^{1 / 2}\right)\right]}{2\left(y-y_{1}\right)\left[\mathcal{K}_{1}\left(2\left(y-y_{1}\right)^{1 / 2}\right)\right]^{2}}
$$

Defining

$$
g_{2}(y)=\frac{\left[\mathcal{K}_{0}\left(2\left(y-y_{1}\right)^{1 / 2}\right)\right]}{2\left(y-y_{1}\right)\left[\mathcal{K}_{1}\left(2\left(y-y_{1}\right)^{1 / 2}\right)\right]^{2}}
$$


we can write

$$
\begin{aligned}
& G(\rho, y)=g_{1}(y)+\left[\frac{g_{1}(y)+g_{2}(y)}{2}\right] \rho+\cdots \\
& I \sim \int_{0}^{\infty} \pi e^{-\rho t} e^{-i k y t}\left[\frac{\rho}{k}-\frac{\rho^{2}}{2 k^{2}}+\cdots\right] \sum_{n=0}^{\infty} \frac{(-\rho)^{n}}{(i k y)^{n+1}} \\
& \\
& {\left[g_{1}(y)+\frac{\left(g_{1}(y)+g_{2}(y)\right)}{2} \rho+\cdots\right] \mathrm{d} \rho}
\end{aligned}
$$

As seen an Appendix A each power of $\rho$ in the integrand gives an inverse power of $t$ in the resulting expression after integration. The integral in (C.5) is the infinite sum of integrals of the form

$$
\int_{0}^{\infty} \rho^{n} e^{-\rho t} \mathrm{~d} \rho \sim O\left(\frac{1}{t^{n+1}}\right)
$$

After integration we obtain an asymptotic series. For $t \gg 1$ we only need the first term or two. In the series (C.1) for $\frac{1}{-\rho-i k y}$ each power of $\rho$ is devided by a power of $y$. Since $y \ll 1$ we need to retain all the terms in this series. We can approximate the other two series (C.2) and (C.4) by their first terms. Applying of Watson's lemma (see, for example, Nayfeh, 1981) to calculate the integral (C.5) gives the result

$$
I \sim-\frac{1}{2} e^{-i k y t} g_{1}(y) \frac{1}{k t} \sum_{n=0}^{\infty} \frac{i^{n+1}(n+1) !}{(k y t)^{n+1}}
$$

It can be verified that the first neglected term in this expression is of order $t^{-3}$.

We use a similar procedure to evaluate $I^{\prime}$ for the contribution from the branch point $s=-i k y_{1}$. In (A.7), the denominator can be approximated by using $\mathcal{K}_{1}(z) \sim$ 
$\frac{1}{z}$. This time there is no factor of $y$ in the denominator so we only need the first term of each series. After taking the first term and integrating we obtain the same solution that we have in the outer region which is of the form

$$
I^{\prime} \sim e^{-i k y_{1} t} O\left(t^{-1}\right)+O\left(t^{-2}\right)
$$




\section{Bibliography}

[1] Abramowitz, M., Stegun, I.A., 1964, Handbook of mathematical functions with formulas, graphs and mathematical tables, Nat. Bur. Stands, 1046 pp.

[2] Béland, M., 1976, Numerical study of the nonlinear Rossby wave critical level development in a barotropic zonal flow, J. Atmos. Sci., 33, 2066-2078.

[3] Benney, D.J., Bergeron, R.F., 1969, A new class of nonlinear waves in parallel flows, Stud. Appl. Math., 48, 181-204.

[4] Booker, J.R., Bretherton, F.P., 1967, The critical layer for gravity waves in a shear flow, J. Fluid Mech., 27, 513-539.

[5] Brown, S.N., Stewartson, K., 1982, On the nonlinear of reflextion of a gravity wave at a critical level, Part 3, J. Fluid Mech.,115, 231-250.

[6] Brunet, G., Haynes, P.H., 1996, Low-latitude reflection of Rossby wave trains, J. Atmos. Sci., 53, 482-496.

[7] Campbell, L.J., 2004, Wave-mean-flow interactions in a forced Rossby wave packet critical layer, Stud. Appl. Math., 112, 39-85. 
[8] Campbell, L.J., Maslowe, S.A., 1998, Forced Rossby wave packets in barotropic shear flows with critical layers, Dyn. Atmos. Oceans, 28, 9-37.

[9] Campbell, L.J., Maslowe, S.A., 2003, Nonlinear critical layer evolution of a forced gravity wave packet, J. Fluid Mech., 493, 151-179.

[10] Davis, R.E., 1969, On the high Reynolds number flow over a wavy boundary, J. Fluid Mech., 36, 337-346.

[11] Dickinson, R.E., 1970, Development of a Rossby wave critical level, J. Atmos. Sci., 27, 627-633.

[12] Enomoto, T., Matsuda, Y., 1999, Rossby wave packet propagation in a zonallyvarying basic flow, Tellus, Rossby-100 special issue, 51A, 588-602.

[13] Hare, F.K., 1968, The Arctic, Quart. J. Roy. Met. Soc., 94, 439-459.

[14] Holton, J.R., 1975, The Dynamic meteorology of the stratosphere and mesosphere, Amer. Met. Society, 218 pp.

[15] Hughes, C.W., 1996, The Antarctic Circumpolar Current as a waveguide for Rossby waves, J. Phys. Oceanogr., 26, 1375-1387.

[16] Kundu, P.K., Cohen, I.M., 2002, Fluid Mechanics, San Diego : Academic Press, $638 \mathrm{pp}$.

[17] Lin, C.C., 1966, The theory of hydrodynamic stability, Cambridge Univ. Press, 155 pp. 
[18] Lindzen, R.S., Holton, J.R., 1968, A theory of the quasi-biennial oscillation, $J$. Atmos. Sci., 25, 1095-1107.

[19] Matsuno, T., 1971, A dynamical model of the stratospheric sudden warming, J. Atmos. Sci., 28, 1479-1494.

[20] Molteni, F., Tibaldi, S., Palmer, T.N., 1990, Regimes in the wintertime circulation over the northern extratropics, I: Observational evidence, Q. J.R. Meteorol. Soc., 116, 31-67.

[21] Nayfeh, A.H., 1981, Introduction to perturbation techniques, Wiley-Interscience publications, $519 \mathrm{pp}$.

[22] Pedlosky, J., 1979, Geophysical fluid dynamics, Springer-Verlag, 624 pp.

[23] Stewartson, K., 1978, The evolution of the critical layer of a Rossby wave, Geophys. Astrophys. Fluid Dyn., 9, 185-200.

[24] Warn, T., Warn, H., 1976, On the development of a Rossby wave critical level, J. Atmos. Sci., 33, 2021-2024.

[25] Warn, T., Warn, H., 1978, The evolution of a nonlinear critical level, Stud. Appl. Math., 59, 37-71. 